\title{
Affleck-Dine baryogenesis just after inflation
}

\author{
Masaki Yamada \\ ICRR, The University of Tokyo, Kashiwa, Chiba 277-8582, Japan; \\ Kavli IPMU (WPI), UTIAS, The University of Tokyo, Kashiwa, Chiba 277-8583, Japan, \\ and Deutsches Elektronen-Synchrotron DESY, 22607 Hamburg, Germany
}

(Received 7 December 2015; published 20 April 2016)

\begin{abstract}
We propose a new scenario of Affleck-Dine baryogenesis where a flat direction in the MSSM generates $B-L$ asymmetry just after the end of inflation. The resulting amount of baryon asymmetry is independent of low-energy supersymmetric models but is dependent on inflation models. We consider the hybrid and chaotic inflation models and find that reheating temperature is required to be higher than that in the conventional scenario of Affleck-Dine baryogenesis. In particular, nonthermal gravitino-overproduction problem is naturally avoided in the hybrid inflation model. Our results imply that Affleck-Dine baryogenesis can be realized in a broader range of supersymmetry and inflation models than expected in the literature.
\end{abstract}

DOI: 10.1103/PhysRevD.93.083516

\section{INTRODUCTION}

The big bang theory is successful in explaining the expansion of the Universe, the cosmic microwave background (CMB), and light element abundances. It requires the baryon-to-entropy ratio of order $10^{-10}$ as an initial condition at a temperature above $1 \mathrm{MeV}$. When we consider the earlier Universe, there is an era of exponential expansion, called inflation, which solves cosmological problems related to the initial conditions of the Universe, such as the horizon problem, flatness problem, and origin of large scale structure. However, baryon asymmetry is washed out by inflation, so that we need a mechanism to generate the observed amount of baryon asymmetry after inflation.

In supersymmetric (SUSY) theories, baryon asymmetry can be generated by Affleck-Dine baryogenesis (ADBG) using a $B-L$ charged flat direction called an AD field $[1,2]$. The $A D$ field is assumed to have a negative effective mass term, called a Hubble-induced mass term, due to a finite energy density of the Universe via supergravity effects, which implies that it obtains a large vacuum expectation value (VEV) during and after inflation. As the energy density of the Universe decreases, the effective mass decreases. Eventually, the effective mass becomes comparable to the soft mass of the AD field, and then the $\mathrm{AD}$ field starts to oscillate around the origin of its potential. At the same time, its phase direction is kicked by its A-term potential. Since the $B-L$ number density is proportional to the phase velocity of the AD field, the $B-L$ asymmetry is generated through this dynamics. Finally, the coherent oscillation of the AD field decays and dissipates into the thermal plasma and the $B-L$ asymmetry is converted to the desired baryon asymmetry through the sphaleron effects $[3,4]$. There are many applications of ADBG (e.g., Refs. [5-11]). It could solve the baryon-DM coincidence problem [12-20] and the moduli problem [21-27].
The mechanism can also be used to generate asymmetry in dark sector [28-30]. Inflaton may play a role of the AD field in non-SUSY models [31-33].

As mentioned above, the AD field obtains a Hubbleinduced mass due to the finite energy density of the Universe during and after inflation (see Refs. [34-37] for recent works on Hubble-induced terms.) In the conventional scenario of ADBG, the sign of the Hubble-induced mass term is assumed to be negative during and after inflation. However, the sign of the Hubble-induced mass term can change after inflation because the source of the energy density of the Universe generically changes after inflation. In this paper, we investigate a new scenario that the $\mathrm{AD}$ field obtains a negative Hubble-induced mass term during inflation while it obtains a positive one after inflation. ${ }^{1,2}$ In this case, the AD field starts to oscillate around the origin of the potential due to the positive Hubble-induced mass term just after the end of inflation. At the same time, its phase direction is kicked by an A-term and $B-L$ asymmetry is generated. We calculate the produced amount of baryon asymmetry and show that it can be consistent with that observed.

The whole scenario is much simpler than the conventional scenario of ADBG. This is because the dynamics of the AD field is determined only by the Hubble-induced terms and the low-energy potential of the AD field does not

\footnotetext{
${ }^{1}$ A similar scenario has been considered in the case of D-term inflation models in Refs. [20,38], where the Hubble-induced mass is absent during D-term inflation and arises with a positive coefficient after inflation. In this paper, we focus on F-term hybrid and chaotic inflation models.

${ }^{2}$ The opposite case, where the Hubble-induced mass term is positive during inflation and is negative after inflation, has been considered in Refs. [39,40]. Although $B-L$ asymmetry cannot be generated via the dynamics of the flat direction, topological defects form after inflation and emit gravitational waves.
} 
affect the resulting $B-L$ asymmetry. This means that the scenario and our calculations in this paper can be applied to many SUSY models, including gravity-mediated and gauge-mediated SUSY breaking models. In particular, the scenario does not result in the formation of nontopological solitons called Q-balls even in gauge-mediated SUSY breaking models $[12,41-44]$. This is one of the advantages of our scenario because Q-balls are sometimes problematic due to their long lifetime. In addition, thermal effect on the dynamics of the AD field can be neglected in our scenario because it starts to oscillate before thermal plasma grows. This is the case even for so-called $L H_{u}$ flat direction. However, the resulting $B-L$ asymmetry depends on the energy scale of inflation because the dynamics of the AD field is determined by Hubble-induced terms. In particular, the A-term depends on inflation models, so that we need to calculate $B-L$ asymmetry for each inflation model. Since the resulting $B-L$ asymmetry depends on parameters in inflaton sector, we could check the consistency of the scenario by observing predictions of inflation models, such as the spectral index and tensor-to-scalar ratio.

This paper is organized as follows. In the next section, we briefly review the conventional scenarios of ADBG. Then we consider our scenario of ADBG in the case that the AD field obtains a positive Hubble-induced mass term after inflation. We first overview the scenario in Sec. III. Then we apply it to a hybrid inflation model in Sec. IV and a chaotic inflation model in Sec. V. Finally, we conclude in Sec. VI.

\section{CONVENTIONAL SCENARIO OF ADBG}

In this section, we review the conventional scenario of ADBG to clarify the difference from our scenario explained in the subsequent sections.

\section{A. Preliminary}

In SUSY theories, there are SUSY partners of quarks and leptons, called squarks and sleptons, which are complex scalar fields carrying $B-L$ charges. Let us consider one of them and denote it as $\phi$. When we write its $B-L$ charge as $q$, the number density of $B-L$ asymmetry associated with $\phi$ is written as

$$
n_{B-L}=i q\left(\dot{\phi}^{*} \phi-\phi^{*} \dot{\phi}\right)=2 q \operatorname{Im}\left[\phi^{*} \dot{\phi}\right] .
$$

This implies that we can obtain a large amount of $B-L$ asymmetry when the field $\phi$ rotates in the complex plane with a large amplitude. Thus we focus on a $B-L$ charged scalar field that has a very flat potential. In SUSY theories, there are two types of potentials for scalar fields: D-term and F-term potentials. Although gauged scalar fields have D-term potentials, it is known that D-terms are cancelled for gauge-singlet combinations of scalar fields. For example, when the field $\phi$ consists of the following combination, D-term potentials are cancelled:
$\left(u^{c}\right)_{i}^{R}=\frac{1}{\sqrt{3}} \phi, \quad\left(d^{c}\right)_{j}^{G}=\frac{1}{\sqrt{3}} \phi, \quad\left(d^{c}\right)_{k}^{B}=\frac{1}{\sqrt{3}} \phi$,

where the upper indices represent color and the lower ones represent flavors $(j \neq k)$. The fields $u^{c}$ and $d^{c}$ are $u$-type and $d$-type right-handed squarks, respectively. This D-flat direction is sometimes called $u^{c} d^{c} d^{c}$ flat direction. The following combination is another famous example of flat directions called $L H_{u}$ flat direction [45]:

$$
L_{i}=\frac{1}{\sqrt{2}}\left(\begin{array}{l}
0 \\
\phi
\end{array}\right), \quad H_{u}=\frac{1}{\sqrt{2}}\left(\begin{array}{l}
\phi \\
0
\end{array}\right)
$$

where $L$ and $H_{u}$ are left-handed slepton and up-type Higgs, respectively. F-term potentials are determined by superpotential $W$ as

$$
V_{F}(\phi)=\left|\frac{\partial W}{\partial \phi}\right|^{2}
$$

In the minimal SUSY Standard Model (MSSM), the superpotential is given by

$W^{(\mathrm{MSSM})}=y_{u} Q H_{u} u^{c}-y_{d} Q H_{d} d^{c}-y_{e} L H_{d} e^{c}+\mu H_{u} H_{d}$,

within the renormalizable level, where we omit flavor indices. Here we implicitly assume R-parity conservation to avoid disastrous proton decay. Fortunately, many D-flat directions, including $u^{c} d^{c} d^{c}$ flat direction, have no F-term potential within the renormalizable level. The D- and F-flat directions with nonzero $B-L$ charge is listed in Table. I $[46] .^{3}$ It is expected that the dynamics of such a flat direction can generate a large amount of $B-L$ asymmetry.

In low energy, the AD field obtains soft terms coming from the low-energy SUSY breaking effect. In this section, we consider gravity-mediated SUSY breaking models for simplicity. Note that the conventional scenario of ADBG depends on mediation models, ${ }^{4}$ but our scenario does not as explained in the subsequent sections. We write soft terms of the $\mathrm{AD}$ field as

\footnotetext{
${ }^{3}$ Although $L H_{u}$ flat direction has a potential coming from the Higgs $\mu$-term, it is assumed that $\mu$ is of order the soft mass scale and absorb it to the meaning of $m_{\phi}$ [see Eq. (6)].

${ }^{4}$ When we consider a SUSY model with a gauge mediated SUSY breaking effect, the soft mass of the AD field is suppressed for a VEV larger than the messenger scale [47]. In this case, we have to take into account the formation of nontopological solitons called Q-balls [12,41-44]. The baryon number should be released from Q-balls to explain the observed amount of baryon asymmetry and the scenario is completely different from the one explained in this section $[13,16,18,19]$.
} 
TABLE I. Flat directions in the MSSM and $B-L$ charges [46].

\begin{tabular}{lc}
\hline \hline Flat directions & $B-L$ \\
\hline$L H_{u}$ & -1 \\
$u^{c} d^{c} d^{c}$ & -1 \\
$L L e^{c}$ & -1 \\
$Q d^{c} L$ & -1 \\
$d^{c} d^{c} d^{c} L L$ & -3 \\
$u^{c} u^{c} u^{c} e^{c} e^{c}$ & 1 \\
$Q u^{c} Q u^{c} e^{c}$ & 1 \\
$Q Q Q Q u^{c}$ & 1 \\
$(Q Q Q)_{4} L L L e^{c}$ & -1 \\
$u^{c} u^{c} d^{c} Q d^{c} Q d^{c}$ & -1 \\
\hline \hline
\end{tabular}

$$
V_{\text {soft }}=m_{\phi}^{2}|\phi|^{2}+a m_{3 / 2} W^{(\mathrm{AD})}+\text { c.c. }
$$

where $m_{\phi}\left(\simeq m_{3 / 2}\right)$ is the soft mass of the AD field, $m_{3 / 2}$ is gravitino mass, and $a(=\mathcal{O}(1))$ is a constant. We can assume $a=a^{*}$ without loss of generality. The higherdimensional superpotential of the $\mathrm{AD}$ field $W^{(\mathrm{AD})}$ is determined below.

During and after inflation, the AD field obtains effective potentials from the energy density of inflaton $I$ via supergravity effects. In supergravity, the potential of scalar fields is determined by

$V_{\mathrm{SUGRA}}=e^{K / M_{\mathrm{Pl}}^{2}}\left[\left(D_{i} W\right) K^{i \bar{j}}\left(D_{j} W\right)^{*}-\frac{3}{M_{\mathrm{Pl}}^{2}}|W|^{2}\right]$,

where $K$ is a Kähler potential and $D_{i} W \equiv W_{i}+K_{i} W / M_{\mathrm{Pl}}^{2}$. The subscripts represent the derivatives with respect to corresponding fields, e.g., $W_{i}=\partial W / \partial \phi$ for $i=\phi$, and $K^{i \bar{j}}$ is defined by the inverse of $K_{i \bar{j}}$. We introduce an inflaton $I$ with a Kähler potential of

$$
K=|\phi|^{2}+|I|^{2}+\frac{c}{M_{\mathrm{Pl}}^{2}}|\phi|^{2}|I|^{2},
$$

where $c$ is an $O(1)$ constant. We assume that the F-term potential of $I$ drives inflation and satisfies $\left|W_{I}\right|^{2} \simeq$ $3 H_{\text {inf }}^{2} M_{\mathrm{Pl}}^{2}$, where $H_{\text {inf }}$ is the Hubble parameter during inflation. The supergravity potential of Eq. (7) includes the following interaction:

$$
\begin{gathered}
V \supset \exp \left(\frac{K}{M_{\mathrm{Pl}}^{2}}\right) W_{I}\left(K^{I \bar{I}}\right)^{-1} W_{I}^{*} \\
\simeq\left|F_{I}\right|^{2}\left(1+(1-c)|\phi|^{2}\right),
\end{gathered}
$$

where we assume $\langle\phi\rangle,\langle I\rangle \ll M_{\mathrm{Pl}}$ and neglect irrelevant higher-dimensional terms. Thus the AD field $\phi$ obtains an effective mass term of order the Hubble parameter during inflation:

$$
\begin{gathered}
V \supset c_{H} H_{\mathrm{inf}}^{2}|\phi|^{2} \\
c_{H}=-3(c-1) .
\end{gathered}
$$

This is called a Hubble-induced mass term. ${ }^{5}$

After inflation ends, the inflaton starts to oscillate around the potential minimum and its oscillation energy dominates the Universe. During this inflaton-oscillation dominated era, the Hubble-induced mass comes also from higherdimensional kinetic interactions, which are determined by the Kähler potential as

$$
\mathcal{L}_{\text {kin }}=K_{i \bar{j}} \partial_{\mu} \varphi^{i} \partial^{\mu} \varphi^{* j},
$$

where $\varphi_{i}$ generically represents the fields of $\phi$ and $I$. There is a kinetic interaction of

$$
\mathcal{L}_{\text {kin }} \supset K_{I \bar{I}}|\dot{I}|^{2} \supset \frac{c}{M_{\mathrm{Pl}}^{2}}|\dot{I}|^{2}|\phi|^{2} .
$$

A typical time scale of the dynamics of the AD field is at most of order the Hubble parameter as shown below. That of inflaton is the curvature of its potential, which is larger than the Hubble parameter during inflaton-oscillation dominated era. Thus we can take a time average over the inflaton-oscillation time scale to investigate the dynamics of the AD field. Assuming that the inflaton oscillates in a quadratic potential after inflation, we obtain an effective Hubble-induced mass for $\phi$ after inflation:

$$
\begin{aligned}
& V_{H}=c_{H} H^{2}(t)|\phi|^{2} \\
& c_{H}=-3\left(c-\frac{1}{2}\right),
\end{aligned}
$$

where we use the Virial theorem and include the contribution from the F-term potential. ${ }^{6}$

In the conventional scenario, $c_{H}$ is assumed to be negative during and after inflation. This means that the AD field has a large tachyonic mass and obtains a large VEV during the time of $H(t) \gtrsim m_{\phi}$. Since the AD field has a large $\mathrm{VEV}$, we have to take into account nonrenormalizable terms to investigate its dynamics. Although the superpotential of the AD field is absent within the renormalizable level, it may have a higher-dimensional superpotential such as [52]

\footnotetext{
${ }^{5}$ There is sometimes a Hubble-induced A-term during inflation, but it is not the case in general (see Ref. [48]).

${ }^{6}$ Inflation may be driven by a D-term potential of inflaton. In this case, the Hubble-induced mass is absent during inflation but the AD field stays at a nonzero VEV due to the Hubble-friction effect [49-51]. The inflaton obtains nonzero F-term after inflation ends, so that the AD field obtains a Hubble-induced mass during the inflaton oscillation dominated era. Thus the scenario of ADBG and resulting $B-L$ asymmetry are the same with the ones in F-term inflation.
} 


$$
W^{(\mathrm{AD})}=\lambda \frac{\phi^{n}}{n M_{\mathrm{Pl}}^{n-3}},
$$

where $n(\geq 4)$ is an integer depending on flat directions and $M_{\mathrm{Pl}}\left(\simeq 2.4 \times 10^{18} \mathrm{GeV}\right)$ is the reduced Planck scale. For example, since the neutrinos have nonzero masses (denoted as $m_{\nu_{i}}$ ), we introduce a superpotential of

$$
\begin{aligned}
W^{\left(L H_{u}\right)} & =\frac{m_{\nu_{i}}}{2\left\langle H_{u}\right\rangle^{2}}\left(L_{i} H_{u}\right)^{2}, \\
& \equiv \frac{\lambda}{4 M_{\mathrm{Pl}}} \phi^{4} \quad \text { for } \frac{\phi^{2}}{2}=L H_{u},
\end{aligned}
$$

where $\left\langle H_{u}\right\rangle=\sin \beta \times 174 \mathrm{GeV}$ and $\tan \beta \equiv\left\langle H_{u}\right\rangle /\left\langle H_{d}\right\rangle$. Thus the $\mathrm{LH}_{u}$ flat direction corresponds to the case of $n=4$ in Eq. (17). We can also write a superpotential of $\left(u^{c} d^{c} d^{c}\right)^{2}$, so that $n=6$ for the $u^{c} d^{c} d^{c}$ flat direction. The superpotential leads to an F-term potential of $\phi$ as

$$
V_{F}(\phi)=\lambda^{2} \frac{|\phi|^{2 n-2}}{M_{\mathrm{Pl}}^{2 n-6}},
$$

where we neglect irrelevant higher-dimensional terms in the supergravity potential.

\section{B. Case without thermal effects}

Let us explain the dynamics of the AD field and calculate $B-L$ asymmetry. In this section, we neglect thermal log potential, which is explained and introduced in the next subsection.

As explained in the previous subsection, the potential of the $\mathrm{AD}$ field is given by

$$
\begin{aligned}
V(\phi)= & V_{\text {soft }}+V_{H}+V_{F} \\
= & m_{\phi}^{2}|\phi|^{2}+\left(a m_{3 / 2} \lambda \frac{\phi^{n}}{n M_{\mathrm{Pl}}^{n-3}}+\text { c.c. }\right) \\
& +c_{H} H^{2}(t)|\phi|^{2}+\lambda^{2} \frac{|\phi|^{2 n-2}}{M_{\mathrm{Pl}}^{2 n-6}},
\end{aligned}
$$

during the inflaton-oscillation dominated era. When we decompose the $\mathrm{AD}$ field as $\phi=\varphi e^{i \theta} / \sqrt{2}$, the equations of motion are written as

$$
\begin{gathered}
\ddot{\varphi}+3 H \dot{\varphi}-\dot{\theta}^{2} \varphi+\frac{\partial V(\varphi)}{\partial \varphi}=0 \\
\ddot{\theta}+3 H \dot{\theta}+2 \frac{\dot{\varphi}}{\varphi} \dot{\theta}+\frac{\partial V}{\partial \theta}=0
\end{gathered}
$$

where $H=2 / 3 t$ during the inflaton-oscillation dominated era. Note that the phase direction has a Hubble-friction term $(3 H \dot{\theta})$.
The coefficient $c_{H}$ is assumed to be negative in the conventional scenario of ADBG. In this case, the AD field has a tachyonic mass, so that it obtains a large VEV. The $\mathrm{VEV}$ of the AD field at the potential minimum is given by

$$
\langle|\phi|\rangle \simeq\left(\frac{\left|c_{H}\right| H^{2}(t) M_{\mathrm{Pl}}^{2 n-6}}{\lambda^{2}(n-1)}\right)^{1 /(2 n-4)}
$$

for $H(t) \gtrsim m_{\phi}$. The $\mathrm{AD}$ field follows this potential minimum.

The phase of the flat direction stays at a certain phase due to the Hubble friction term. We denote the initial phase of the $\mathrm{AD}$ field as $\theta_{0}$, which is expected to be of order unity. When the Hubble parameter decreases to $m_{\phi}$, the potential of the AD field is dominated by the soft mass term and it starts to oscillate around the origin of the potential. Here we denote the Hubble parameter at the time of beginning of oscillation as $H_{\mathrm{osc}}$ :

$$
H_{\mathrm{osc}} \simeq \frac{m_{\phi}}{\sqrt{\left|c_{H}\right|}} .
$$

The VEV of the AD field at that time is given by

$$
\phi_{\mathrm{osc}} \simeq\left(\frac{\left|c_{H}\right| H_{\mathrm{osc}}^{2} M_{\mathrm{Pl}}^{2 n-6}}{\lambda^{2}(n-1)}\right)^{1 /(2 n-4)} .
$$

At the same time, its phase direction is kicked by the Aterm, so that it starts to rotate in the phase space. This is the dynamics that generates the $B-L$ asymmetry [see Eq. (1)]. The evolution of equation for the $B-L$ number density is written as

$$
\dot{n}_{B-L}+3 H n_{B-L}=-q \varphi^{2}\left(\frac{\partial V}{\partial \theta}\right) \text {, }
$$

where $q$ denotes the $B-L$ charge of the AD field. We semianalytically and numerically solve this equation and obtain

$$
\begin{aligned}
& a^{3} n_{B-L}(t)=-\int \mathrm{d} t q a^{3}(t) \varphi^{2} \frac{\partial V}{\partial \theta} \\
& \equiv \epsilon q H_{\mathrm{osc}} \phi_{\mathrm{osc}}^{2} a^{3}\left(t_{\mathrm{osc}}\right) \\
& \epsilon \simeq(2-4) \times \frac{a}{\sqrt{n-1}(1+(n-4) /(n-2))} \frac{m_{3 / 2}}{m_{\phi}} \\
& \times \sin \left(n \theta_{0}\right) \quad \text { for } \epsilon \lesssim 1,
\end{aligned}
$$

where we assume $c_{H}=-1$ in the last line. We define the ellipticity parameter $\epsilon(\leq 1)$ which represents the efficiency of baryogenesis. Since the $B-L$ number density has to be smaller than that of the total AD field times $B-L$ charge $q$, $\epsilon$ is at most unity. We have numerically solved the equation of motion for $\phi$ and have obtained the numerical factor of 

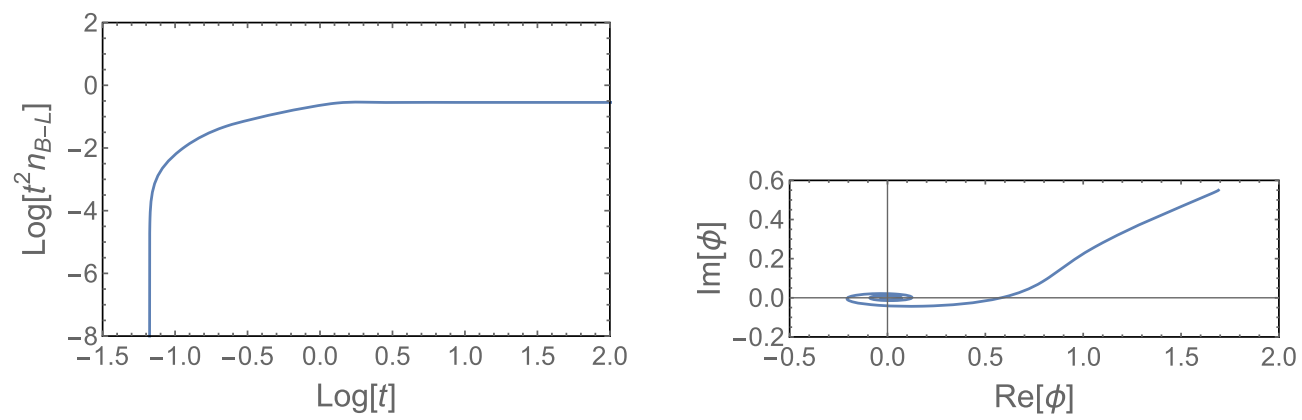

FIG. 1. Evolution of $B-L$ number density in a comoving volume (left panel) and the AD field (right panel) in the conventional scenario of ADBG. We set $n=6, c_{H}=-1, a m_{3 / 2} / m_{\phi}=-1$, and $\theta_{0}=\pi / 10$. The dimensionfull quantities are rescaled such as $t \rightarrow t / m_{\phi}$ and $\phi \rightarrow \phi /\langle|\phi|\rangle_{t=H_{\text {osc }}^{-1}}$.

(2-4) in Eq. (31) for $c_{H}=-1$ and $\epsilon \lesssim 1$. One of the numerical results is shown in Fig. 1 , where we set $n=6$, $c_{H}=-1, a m_{3 / 2} / m_{\phi}=-1$, and $\theta_{0}=\pi / 10$. One can see that the phase direction is kicked and the $B-L$ asymmetry is generated at $t \sim m_{\phi}^{-1} \simeq H_{\mathrm{osc}}^{-1}$. The amplitude of the flat direction decreases as time evolves due to the Hubble expansion and the $B-L$ breaking effect (i.e., the A-term) becomes irrelevant soon after the oscillation. Thus, the generated $B-L$ asymmetry within a comoving volume is conserved soon after the AD field starts to oscillate as one can see in Fig. 1.

Then, the oscillating AD field decays and dissipates into radiation [53] and the sphaleron effect relates the $B-L$ asymmetry to the baryon asymmetry $[3,4]{ }^{7}$ Since the sphaleron process is in thermal equilibrium, the resulting baryon asymmetry is related to the $B-L$ asymmetry such as [54]

$$
n_{b} \simeq \frac{8}{23} n_{B-L}
$$

We can calculate the resulting baryon-to-entropy ratio $Y_{b}$ such as

$$
\begin{aligned}
Y_{b} & \left.\equiv \frac{n_{b}}{s} \simeq \frac{8}{23} \frac{n_{B-L}}{s}\right|_{\mathrm{RH}} \\
& \left.\simeq \frac{8}{23} \frac{3 T_{\mathrm{RH}} n_{B-L}}{4 \rho_{\mathrm{inf}}}\right|_{\mathrm{osc}} \\
& \simeq \frac{8}{23} \frac{\epsilon q T_{\mathrm{RH}}}{4 H_{\mathrm{osc}}}\left(\frac{\phi_{\mathrm{osc}}}{M_{\mathrm{Pl}}}\right)^{2}
\end{aligned}
$$

\footnotetext{
${ }^{7}$ For simplicity, in this section we assume that Q-balls do not form after ADBG. Note that in our scenario explained in the subsequent sections, Q-balls do not form.
}

$$
\begin{aligned}
\simeq & 1.2 \times 10^{-10} \epsilon q \lambda^{-1 / 2}\left(\frac{T_{\mathrm{RH}}}{100 \mathrm{GeV}}\right) \\
& \times\left(\frac{m_{\phi}}{1 \mathrm{TeV}}\right)^{-1 / 2} \quad \text { for } n=6,
\end{aligned}
$$

where $\rho_{\text {inf }}\left(\simeq 3 H^{2}(t) M_{\mathrm{Pl}}^{2}\right)$ is the energy density of the inflaton and $T_{\mathrm{RH}}$ is reheating temperature. In the last line, we use Eq. (27). The resulting baryon asymmetry can be consistent with the observed baryon asymmetry of $Y_{b}^{(\text {obs })} \simeq$ $8.7 \times 10^{-11}$ [55]. Since we expect $\epsilon q \sim 1$, a relatively low reheating temperature is required to explain the observed amount of baryon asymmetry unless the parameter $\lambda$ is much larger than unity.

\section{Case with thermal effects: $L H_{u}$ flat direction}

In this section, we take into account thermal log potential. It is particularly important for the case of $n=4$, including the case of $L H_{u}$ flat direction.

After inflation ends and before reheating completes, inflaton gradually decays into radiation. Since the energy density of radiation is given by $\rho_{\text {rad }} \simeq(3 / 5) \rho_{\text {inf }} \Gamma_{I} t$, there is a background plasma with a temperature of

$$
T=\left(\frac{36 H(t) \Gamma_{I} M_{\mathrm{Pl}}^{2}}{g_{*}(T) \pi^{2}}\right)^{1 / 4},
$$

where $g_{*}$ is the effective number of relativistic degrees of freedom in the thermal plasma. The decay rate of inflaton $\Gamma_{I}$ is related with the reheating temperature as

$$
T_{\mathrm{RH}} \simeq\left(\frac{90}{g_{*}\left(T_{\mathrm{RH}}\right) \pi^{2}}\right)^{1 / 4} \sqrt{\Gamma_{I} M_{\mathrm{Pl}}} .
$$

Here we explain the origin of the thermal log potential, focusing on $\mathrm{LH}_{u}$ flat direction. The free energy of the thermal plasma $F$ depends on QCD coupling $g_{s}$ in the nextto-leading order as 

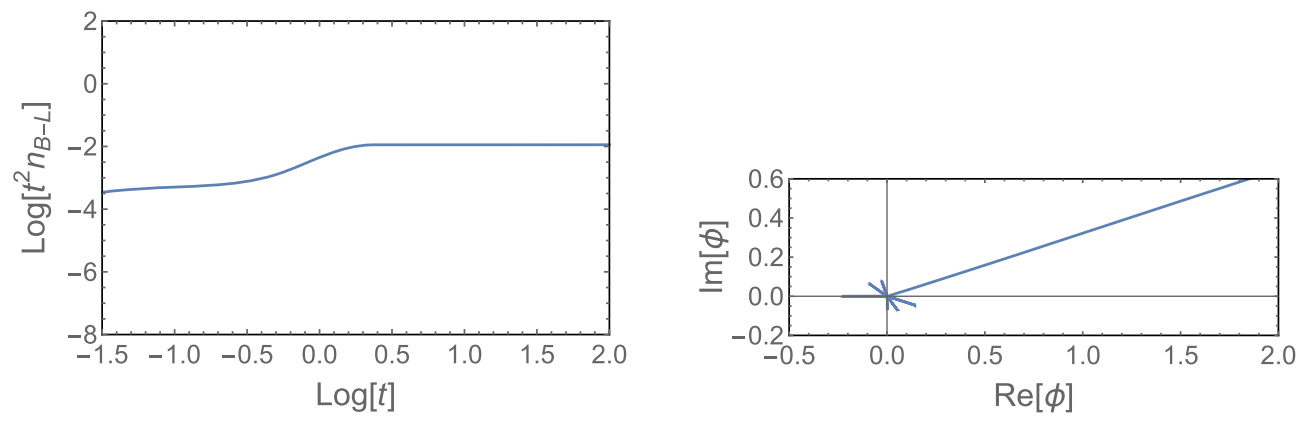

FIG. 2. Evolution of $B-L$ number density in a comoving volume (left panel) and the phase direction of the AD field (right panel) in the conventional scenario of ADBG. We set $c_{H}=-1, a_{H} m_{3 / 2} / H_{\text {osc }}=-0.01$, and $\theta_{0}=\pi / 10$. The dimensionfull parameters are rescaled as $t \rightarrow t / H_{\mathrm{osc}}$ and $\phi \rightarrow \phi /\langle|\phi|\rangle_{t=H_{\mathrm{osc}}^{-1}}$.

$$
F=\frac{3}{8}\left(1+N_{f}^{(\mathrm{th})}\right) g_{s}^{2}(T) T^{4}
$$

where $N_{f}^{(\text {th) }}$ is the number of family in the thermal plasma. Here, the quark multiplets obtain effective masses via the Yukawa interactions when $L H_{u}$ flat direction has a large VEV [see Eq. (5)]. When its VEV is larger than the temperature of the plasma, the renormalization running of $g_{s}$ is affected and its value at the energy scale of $T$ depends on the VEV of $L H_{u}$ flat direction: $g_{s}(T)=$ $g_{s}(T, \phi)$. Therefore the free energy depends on $\phi$ and $L H_{u}$ flat direction acquires a potential depending on temperature. Since the renormalization running has a logarithmic dependence, it is written as $[5,6]$

$$
V_{T}(\phi) \simeq c_{T} \alpha_{s}^{2} T^{4} \log \left(\frac{|\phi|^{2}}{T^{2}}\right),
$$

with $c_{T}=45 / 32$ for $y|\phi| \gg T$, where $\alpha_{s} \equiv g_{s}^{2} / 4 \pi$ and $y$ generically stands for Yukawa couplings for quarks. This is sometimes called thermal log potential.

In the previous subsection, we neglect the thermal potential and the $\mathrm{AD}$ field starts to oscillate around the origin of the potential at $H(t) \simeq m_{\phi} / \sqrt{\left|c_{H}\right|}$. When we take into account the thermal log potential, it starts to oscillate at the time of

$$
H_{\mathrm{osc}} \simeq \operatorname{Max}\left[\frac{m_{\phi}}{\sqrt{\left|c_{H}\right|}}, \sqrt{\phi^{-1} V_{T}^{\prime}}\right] .
$$

Using Eqs. (27) and (37), this can be rewritten as

$$
H_{\mathrm{osc}} \simeq \operatorname{Max}\left[m_{\phi}, 0.6 \alpha_{s} \sqrt{\lambda} T_{\mathrm{RH}}\right],
$$

where we assume $\left|c_{H}\right|=1$ and $n=4$.

We numerically solve the equation of motion for $\phi$ and obtain the ellipticity parameter as

$$
\epsilon=(0.4-3.5) \times a \sin \left(n \theta_{0}\right) \frac{m_{3 / 2}}{H_{\mathrm{osc}}}
$$

$$
\equiv \tilde{\epsilon} \frac{m_{3 / 2}}{H_{\mathrm{osc}}},
$$

where we define $\tilde{\epsilon}$ that is expected to be of order unity. Here we assume $T_{\mathrm{RH}} \gtrsim m_{\phi} /\left(\alpha_{s} \sqrt{\lambda}\right)$, which implies $H_{\mathrm{osc}} \simeq$ $0.6 \alpha_{s} \sqrt{\lambda} T_{\mathrm{RH}}$ [see Eq. (42)]. One of our results is shown in Fig. 2, where we set $c_{H}=1, a m_{3 / 2} / H_{\text {osc }}=-0.01$, and $\theta_{0}=\pi / 10$. The ellipticity parameter $\epsilon$ is much smaller than unity in this numerical calculation, so that the phase direction is kicked slightly. It is difficult to see that the $\mathrm{AD}$ field rotates in the phase space in the right panel of Fig. 2 though it actually does.

The baryon-to-entropy ratio is calculated as

$$
\begin{aligned}
Y_{b} & \simeq \frac{8}{23} \frac{q \tilde{\epsilon} m_{3 / 2}}{4 \alpha_{s} \lambda^{3 / 2} M_{\mathrm{Pl}}} \\
& \simeq 3.7 \times 10^{-10} \tilde{\epsilon}\left(\frac{\lambda}{10^{-4}}\right)^{-3 / 2}\left(\frac{m_{3 / 2}}{1 \mathrm{TeV}}\right),
\end{aligned}
$$

where we assume $T_{\mathrm{RH}} \gtrsim m_{\phi} /\left(\alpha_{s} \sqrt{\lambda}\right), \quad\left|c_{H}\right|=1$, and $\alpha_{s}=0.1$ and use $\epsilon=\tilde{\epsilon} m_{3 / 2} / H_{\text {osc }}$. This result is independent of the reheating temperature [6]. The observed baryon asymmetry can be explained when the coupling $\lambda$ satisfies

$$
\lambda \simeq 2.6 \times 10^{-4}\left(\frac{m_{3 / 2}}{1 \mathrm{TeV}}\right)^{2 / 3},
$$

where we assume $\tilde{\epsilon}=1$. When we identify the AD field as $L H_{u}$ flat direction, this result implies that the lightest lefthanded neutrino has a tiny mass of

$$
m_{\nu} \simeq 1.6 \times 10^{-9} \mathrm{eV}\left(\frac{\lambda}{2.6 \times 10^{-4}}\right)
$$

$$
\simeq 1.6 \times 10^{-9} \mathrm{eV}\left(\frac{m_{3 / 2}}{1 \mathrm{TeV}}\right)^{2 / 3} .
$$




\section{Baryonic isocurvature constraint}

In many cases, the phase direction of the AD field is massless during inflation. This implies that the phase direction has a quantum fluctuation during inflation $[19,48,50,51]$ :

$$
\left|\delta \theta_{0}\right| \simeq \frac{\sqrt{2} H_{\mathrm{inf}}}{2 \pi|\phi|_{\mathrm{inf}}}
$$

Since the resulting baryon asymmetry is related to $\theta_{0}$ [see Eqs. (31) and (43)], ADBG predicts baryonic isocurvature perturbations such as

$$
\mathcal{S}_{b \gamma} \equiv \frac{\delta Y_{B}}{Y_{B}} \simeq n \cot \left(n \theta_{0}\right) \delta \theta_{0}
$$

Since the density perturbations of the CMB are predominantly adiabatic, the baryonic isocurvature perturbation is tightly constrained as [56]

$$
\left|\mathcal{S}_{\mathrm{b} \gamma}\right| \lesssim 5.0 \times 10^{-5}
$$

Therefore, this constraint puts an upper bound on the energy scale of inflation:

$$
H_{\text {inf }} \lesssim 5.3 \times 10^{14} \mathrm{GeV} \frac{\tan \left(n \theta_{0}\right)}{n} \frac{|\phi|_{\text {inf }}}{M_{\mathrm{Pl}}} .
$$

This can be rewritten as

$H_{\text {inf }} \lesssim \begin{cases}1.6 \times 10^{13} \mathrm{GeV}\left(\frac{\lambda}{2.6 \times 10^{-4}}\right)^{-1} & \text { for } n=4 \\ 2.3 \times 10^{12} \mathrm{GeV} \lambda^{-1 / 3} & \text { for } n=6,\end{cases}$

where we use Eq. (25) and assume $\left|c_{H}\right|=1$ and $\tan \left(n \theta_{0}\right)=1$.

\section{AFFLECK-DINE BARYOGENESIS JUST AFTER INFLATION}

In this section, we explain a new scenario of ADBG where the $\mathrm{AD}$ field starts to oscillate around the origin of the potential just after the end of inflation. In general, this scenario is realized when the Kähler potential is give by

$K=|\phi|^{2}+|S|^{2}+|\psi|^{2}+\frac{c_{1}}{M_{\mathrm{Pl}}^{2}}|\phi|^{2}|S|^{2}-\frac{c_{2}}{M_{\mathrm{Pl}}^{2}}|\phi|^{2}|\psi|^{2}$,

where $S$ is the field whose F-term drives inflation and $\psi$ is the field whose oscillation energy dominates the Universe after inflation. Here, we assume that the fields $S$ and $\psi$ are different fields, which is actually the case in hybrid and chaotic inflation models as shown in the subsequent sections.
During inflation, the AD field acquires the Hubbleinduced mass via the F-term potential of the field $S$ as Eq. (10). After inflation ends, the Hubble-induced mass comes also from higher-dimensional kinetic interactions between $\phi$ and $\psi$ as Eq. (16). Therefore, the Hubble induced mass term for the $\mathrm{AD}$ field $\phi$ is given by

$V_{H}=c_{H} H^{2}(t)|\phi|^{2}$

$c_{H}= \begin{cases}-3\left(c_{1}-1\right) & \text { during inflation } \\ 3\left(-(1-r) c_{1}+r c_{2}+\frac{1}{2}\right) & \text { after inflation, }\end{cases}$

where $r(0 \leq r \leq 1)$ is the fraction of the energy density of $\psi$ to the total energy after inflation. Therefore the sign of the Hubble-induced mass term can change after inflation. If its sign continues to be negative after inflation, the conventional scenario of ADBG is realized as we explain in the previous section. In the rest of this paper, we consider the case that the coefficient is negative during inflation and is positive after inflation. In this case, the $\mathrm{AD}$ field starts to oscillate around the origin of the potential just after the end of inflation. In contrast to the conventional scenario of ADBG, the dynamics of its phase direction depends on inflation models, so that the resulting $B-L$ asymmetry depends on parameters in the inflaton sector. In the subsequent sections, we consider hybrid and chaotic inflation models to investigate this scenario and calculate the amount of $B-L$ asymmetry. Before we investigate the detail of the dynamics of the AD field, we explain its rough behavior in this section.

In the above scenario, the dynamics of the $\mathrm{AD}$ field is determined by the potential of

$$
V(\phi)=c_{H} H^{2}(t)|\phi|^{2}+\lambda^{2} \frac{|\phi|^{2 n-2}}{M_{\mathrm{Pl}}^{2 n-6}}+V_{A}(\phi),
$$

where $c_{H}<0$ during inflation and $c_{H}>0$ after inflation. Here we have taken a time average over the inflatonoscillation time scale as we have done in Eq. (22), though in the following we also perform numerical calculation taking into account the motions of fields in inflaton sectors. The A-term potential of $V_{A}$ depends on inflation models and is explicitly derived in the subsequent sections. The lowenergy soft terms of Eq. (6) are irrelevant for the dynamics of the AD field. This makes our calculation simple and independent of low-energy SUSY models. In particular, the resulting $B-L$ asymmetry is independent of how the SUSY breaking effect is mediated to the visible sector.

Here, let us emphasize that the origin of the $B-L$ breaking term is the same with the one in the conventional scenario of ADBG. The AD field has to be charged under $B-L$ symmetry and there should be a $B-L$ breaking term to satisfy one of the Sakharov conditions to generate $B-L$ asymmetry. In our scenario, the $B-L$ breaking term originates from the superpotential of Eq. (17) as in the 
conventional scenario of ADBG. As a result, there are an A-term [see Eq. (6)] and $V_{A}$ [see Eq. (68) or (110)], both of which break $B-L$ symmetry. The former term is used to generate $B-L$ asymmetry in the conventional scenario of ADBG, while the latter one is used in our scenario. Note that the inflaton field should not have a $B-L$ charge because we focus on $B-L$ production via the dynamics of the AD field. This implies that $V_{A}$ is an explicitly $B-L$ breaking term as the A-term is in the conventional scenario of ADBG.

Since we consider the case that $c_{H}<0$ during inflation and $c_{H}>0$ after inflation, the AD field starts to oscillate around the origin just after the end of inflation. At the same time, its phase direction is kicked by an A-term. The origin of the A-term depends on inflation models and thus the resulting $B-L$ asymmetry does. Here we just write generated $B-L$ asymmetry as

$$
\frac{a^{3}(t)}{a^{3}\left(t_{\mathrm{osc}}\right)} n_{B-L}(t) \equiv q \epsilon H_{\mathrm{osc}}|\phi|_{\mathrm{osc}}^{2}
$$

and derive $\epsilon$ in the subsequent sections. The resulting baryon-to-entropy ratio is thus written as

$$
\begin{aligned}
Y_{b} & \left.\simeq \frac{8}{23} \frac{3 T_{\mathrm{RH}} n_{B-L}}{4 \rho_{\mathrm{inf}}}\right|_{\mathrm{osc}} \\
& \simeq \frac{8}{23} \frac{\epsilon q T_{\mathrm{RH}}}{4 H_{\mathrm{osc}}}\left(\frac{\phi_{\mathrm{osc}}}{M_{\mathrm{Pl}}}\right)^{2} .
\end{aligned}
$$

This is the same with Eq. (35) but $H_{\text {osc }}$ is not given by Eqs. (26) and (41). Since the AD field starts to oscillate just after the end of inflation in this scenario, $H_{\mathrm{osc}}$ is given by the Hubble parameter at the end of inflation. Here, let us emphasise differences from the conventional scenario of ADBG. The Hubble parameter at the time of beginning of oscillation $H_{\text {osc }}$ is determined by the energy scale of inflation, not by either $m_{\phi}$ nor $T_{\mathrm{RH}}$ [see Eqs. (26) and (42)]. This is because the flat direction starts to oscillate just after the end of inflation due to the positive Hubble-induced mass term. In addition, $\phi_{\text {osc }}$ depends only on $H_{\text {osc }}$ and $\lambda$ via Eq. (27). Therefore, the resulting $B-L$ asymmetry is independent of parameters in low-energy SUSY models, such as $m_{\phi}$ and $m_{3 / 2}$.

There are some advantages in this scenario. First, as we explain above, the resulting $B-L$ asymmetry is independent of the masses of the AD field and gravitino. The result is also independent of how the SUSY breaking effect is mediated to the visible sector. Second, nontopological solitons, called Q-balls, may form and affect the cosmological scenario after the conventional scenario of ADBG $[12,41-44]$, while they do not form in our scenario. This makes the discussion much simpler. In particular, Q-balls usually form in gauge mediated SUSY breaking models after the conventional scenario of ADBG and they are sometimes problematic in cosmology due to their long lifetime $[19,44]$. Our scenario does not suffer from this problem. Third, the thermal effect on the AD field can be neglected because the AD field starts to oscillate just after the end of inflation and before the thermal plasma grows sufficiently [57]. This also makes calculations simpler. In particular, the thermal log potential can be neglected even for $L H_{u}$ flat direction. Finally, our results imply that ADBG works in a broader range of parameter space. Since the sign of the Hubble-induced mass term cannot be determined by underlying physics, it is equally possible that the sign becomes positive after inflation. In addition, viable parameter regions for some parameters, e.g., the reheating temperature, are different from the ones in the conventional scenario of ADBG. These facts imply that the Affleck-Dine mechanism works well in more cases than expected in the literature.

\section{HYBRID INFLATION}

In this section, we consider our scenario of ADBG in the simplest hybrid inflation model $[58,59]$ and calculate $B-L$ asymmetry. The superpotential in the inflaton sector is given by

$$
W^{(\mathrm{inf})}=\kappa S\left(\psi \bar{\psi}-\mu^{2}\right),
$$

where $S$ is the inflaton, and $\psi$ and $\bar{\psi}$ are waterfall fields. The F-term potentials are thus given as

$$
\left.V_{\text {inf }}\right|_{\text {tree }}=\kappa^{2}\left|\psi \gamma \bar{\psi}-\mu^{2}\right|^{2}+\kappa^{2}|S|^{2}\left(|\psi|^{2}+|\bar{\psi}|^{2}\right) .
$$

The inflaton $S$ is assumed to have a large initial VEV so that the waterfall fields stay at the origin due to effective masses of $\kappa\langle S\rangle$. Then the F-term of $S$ is nonzero and drives inflation, where the energy scale of inflation is given by $3 H_{\mathrm{inf}}^{2} M_{\mathrm{Pl}}^{2} \simeq \kappa^{2} \mu^{4}$. The inflaton $S$ slowly rolls toward the origin due to the 1-loop Coleman-Weinberg potential:

$$
\begin{aligned}
\left.V_{\text {inf }}\right|_{1-\text { loop }}= & \frac{\kappa^{4} \mu^{4}}{32 \pi^{2}}\left[\left(x^{2}+1\right)^{2} \ln \left(x^{2}+1\right)\right. \\
& \left.+\left(x^{2}-1\right)^{2} \ln \left(x^{2}-1\right)-2 x^{4} \ln x^{2}-3\right],
\end{aligned}
$$

where we define $x \equiv|S| / \mu$. Inflation ends when its VEV decreases to the critical value of $S_{c r} \equiv \mu$. The Hubble parameter at the end of inflation is given by

$$
H_{\mathrm{osc}} \simeq H_{\mathrm{inf}} \simeq \frac{\kappa \mu^{2}}{\sqrt{3} M_{\mathrm{Pl}}} .
$$

After that, the waterfall fields as well as the inflaton start to oscillate around the minimum of the potential and their oscillation energy dominates the Universe. Around the minimum of the potential, the masses of inflaton and waterfall fields are given by $\sqrt{2} \kappa \mu$. 
Although the simplest hybrid inflation model predicts an inconsistent spectral index with the observed value, some modifications can make it consistent. For example, we may introduce a higher dimensional Kähler potential for the inflaton to write a small negative mass term, which can result in a consistent spectral index [60,61]. Since our discussion below is not affected at least quantitatively in this modification, we calculate $B-L$ asymmetry in the above simplest model.

\section{A. Dynamics of the AD field}

The inflaton $S$ is identified with the field $S$ in Eq. (55) and the waterfall fields $\psi$ and $\bar{\psi}$ play a role of the field $\psi$ in Eq. (55). Thus the coefficient of the Hubble-induced mass $c_{H}$ can change after inflation. In this subsection, we consider the dynamics of the $\mathrm{AD}$ field in the hybrid inflation model and calculate $B-L$ asymmetry.

Let us first consider the dynamics of the phase direction of the AD field. Using Eq. (7) with the total superpotential of $W^{(\mathrm{AD})}+W^{(\mathrm{inf})}$, we find that there is an A-term potential coming from

$$
\begin{aligned}
& W_{S}^{(\mathrm{inf})} K^{\bar{S} \phi} W_{\bar{\phi}}^{(\mathrm{AD})}+K_{\phi} W^{(\mathrm{inf})}\left(W_{\phi}^{(\mathrm{AD})}\right)^{*}+K_{S} W^{(\mathrm{AD})}\left(W_{S}^{(\mathrm{inf})}\right)^{*} \\
& \quad-3 W^{(\mathrm{inf})}\left(W^{(\mathrm{AD})}\right)^{*}+\text { c.c. }
\end{aligned}
$$

The A-term is written as

$$
\begin{aligned}
V_{A} & =-\left(1-c_{1}-\frac{2}{n}\right) \frac{\kappa \mu^{2} \lambda}{M_{\mathrm{Pl}}^{n-1}} S^{*} \phi^{n}+\text { c.c. } \\
& =-a \frac{H_{\mathrm{inf}}^{2}}{M_{\mathrm{Pl}}}|S||\phi|^{2} \cos \left(\theta_{S}-n \theta_{\phi}\right), \\
a & \equiv-2\left(c_{1}-1+\frac{2}{n}\right) \sqrt{\frac{3\left|c_{H}\right|}{n-1}},
\end{aligned}
$$

where $\theta_{S}$ and $\theta_{\phi}$ are the complex phases of the fields $S$ and $\phi$, respectively. We use Eq. (25) and $H_{\mathrm{inf}}^{2}=\kappa^{2} \mu^{4} / 3 M_{\mathrm{Pl}}^{2}$ in the second line. This is a linear term of the inflaton $S$, so that the slope of the potential should not be larger than that of the Coleman-Weinberg potential [61-63]. Otherwise the inflaton cannot reach the critical VEV and inflation cannot terminate unless we allow a fine-tuning on the initial phase of inflaton. Referring to Ref. [63], we introduce a parameter to describe the relative importance of the two contributions to the slope of the potential:

$$
\begin{aligned}
\xi & \equiv \frac{1}{2}\left(1-c_{1}-\frac{2}{n}\right) \frac{16 \pi^{2}}{\kappa^{3} \ln 2} \frac{\langle|\phi|\rangle^{n}}{\mu M_{\mathrm{Pl}}^{n-1}} \\
& \simeq \frac{8 \pi^{2} a}{3 \ln 2} \frac{\mu\langle|\phi|\rangle^{2}}{\kappa^{2} M_{\mathrm{Pl}}^{3}}
\end{aligned}
$$

which should be smaller than unity so that the inflaton can roll towards the critical value without the fine-tuning. ${ }^{8}$

In the above minimal setup, there is no other term than Eq. (68) that affects the dynamics of the phase directions. Therefore, there is only one massive phase during inflation. For simplicity, let us assume that the inflaton and the $\mathrm{AD}$ field have approximately constant VEVs and $\left(\theta_{S}-n \theta_{\phi}\right) \ll 1$. In this case, the unitary matrix to diagonalize the squared mass matrix for the phase directions is given by

$$
\frac{1}{\sqrt{n^{2}|S|^{2}+|\phi|^{2}}}\left(\begin{array}{ll}
|\phi| & -n|S| \\
n|S| & |\phi|
\end{array}\right)
$$

in the $\left(|S| \theta_{S} / \sqrt{2},|\phi| \theta_{\phi} / \sqrt{2}\right)^{T}$ basis. Thus, the massive direction denoted by $f_{m} \theta_{m}$ can be written as

$$
f_{m} \theta_{m}=\frac{\sqrt{2}|S||\phi|}{\sqrt{n^{2}|S|^{2}+|\phi|^{2}}}\left(\theta_{S}-n \theta_{\phi}\right)
$$

and its mass $m_{\theta_{m}}$ is given by

$$
m_{\theta_{m}}=\sqrt{\frac{a H^{2}}{2} \frac{|\phi|}{M_{\mathrm{Pl}}}\left(\frac{|\phi|}{|S|}+n^{2} \frac{|S|}{|\phi|}\right)} .
$$

If the curvature of the phase direction is larger than the Hubble parameter during inflation, it stays at the minimum of the A-term, i.e., $\theta_{m}=0$, and the phase direction cannot be kicked in the complex plane after inflation. In this case, $B-L$ asymmetry cannot be generated. Thus, we require $m_{\theta_{m}} \ll H$, which can be rewritten as

$$
\begin{gathered}
a|\phi|^{2} \ll|S| M_{\mathrm{Pl}}, \\
a n^{2}|S| \ll M_{\mathrm{Pl}},
\end{gathered}
$$

in order that the phase direction can stay at a different phase from the minimum due to the Hubble friction effect. We denote the initial phase as $\theta_{m}^{\text {ini }}$.

After inflation ends, the $\mathrm{AD}$ field acquires a positive Hubble-induced mass term and starts to oscillate around the origin of the potential. At the same time, the massive phase direction is kicked by the above A-term. Since the radial direction decreases with time due to the Hubble expansion, the A-term is relevant just after the beginning of oscillation. Thus we can estimate the angular velocity of massive phase direction such as

\footnotetext{
${ }^{8}$ When the VEV of the AD field is so large that the parameter $\xi$ becomes of order unity (but below unity), the A-term of Eq. (68) affects inflaton dynamics. As a result, the spectral index can be consistent with the observed value [11].
} 


$$
\dot{\theta}_{m} \approx \frac{m_{\theta_{m}}^{2}}{H} \theta_{m}^{\text {ini }},
$$

[see Eq. (24)]. Using the inverse of the unitary matrix of Eq. (72), we obtain the angular velocity of the phase of the $\mathrm{AD}$ field such as

$$
\begin{aligned}
\dot{\theta}_{\phi} & =\frac{-n|S|}{\sqrt{n^{2}|S|^{2}+|\phi|^{2}} \frac{f_{m}}{\sqrt{2}|\phi|}} \dot{\theta}_{m} \\
& \approx \frac{m_{\theta_{m}}^{2}}{H} \frac{-n|S|}{\sqrt{n^{2}|S|^{2}+|\phi|^{2}}} \frac{f_{m} \theta_{m}^{\text {ini }}}{\sqrt{2}|\phi|} \\
& =\frac{m_{\theta_{m}}^{2}}{H} \frac{-n|S|}{\sqrt{n^{2}|S|^{2}+|\phi|^{2}}} \frac{|S|}{\sqrt{n^{2}|S|^{2}+|\phi|^{2}}}\left(\theta_{S}-n \theta_{\phi}\right)^{\text {ini }} \\
& =-\frac{a n}{2} \frac{|S|}{M_{\mathrm{Pl}}} H\left(\theta_{S}-n \theta_{\phi}\right)^{\mathrm{ini}} .
\end{aligned}
$$

Since we take a time average over the period of an inflatonoscillation time scale, kinetic interactions between $\phi$ and the waterfall fields are effectively taken into account in $V_{H} \cdot{ }^{9}$ Thus we can use Eqs. (23) and (24) as the equations of motion for $\phi$, so that we can also use Eq. (28) to calculate $B-L$ asymmetry. Thus we obtain

$$
\begin{aligned}
\frac{a^{3}(t)}{a^{3}\left(t_{\mathrm{osc}}\right)} n_{B-L}(t) & =\left.2 \dot{\theta}_{\phi}|\phi|^{2}\right|_{\mathrm{osc}} \\
& \equiv \epsilon q H_{\mathrm{osc}} \phi^{2} \\
\epsilon & \equiv \tilde{\epsilon} \frac{S_{\mathrm{cr}}}{M_{\mathrm{Pl}}} \\
\tilde{\epsilon} & \simeq(0.1-0.2) \text { an } \sin \left(n \theta_{\phi}-\theta_{S}\right)_{\mathrm{osc}},
\end{aligned}
$$

where we define $\tilde{\epsilon}$ which is expected to be of order unity. The numerical factor of $(0.1-0.2)$ is determined from our numerical calculations explained below. Note that the resulting ellipticity parameter $\epsilon$ is consistent with a naive estimation of $\epsilon \sim V_{A}^{\prime} / \phi H_{\mathrm{osc}}^{2}{ }^{10}$ The ellipticity parameter $\epsilon$, which describes the efficiency of baryogenesis, is much smaller than unity because of the condition of Eq. (76). This is because the phase direction of the AD field is kicked by the A-term that is suppressed by the VEV of the inflaton.

\footnotetext{
${ }^{9}$ We have also performed numerical calculations to solve the equations of motion for $\phi, S$, and $\psi$ without taking the time average. These results are consistent with each other as we explain below.

${ }^{10}$ We implicitly assume that $\left(S_{c r} / M_{\mathrm{Pl}}\right) \gtrsim m_{3 / 2} / H_{\text {osc }}$ so that we can neglect an A-term of $m_{3 / 2} W_{\phi}$ [see Eq. (6)]. Otherwise $\epsilon$ may be of order $m_{3 / 2} / H_{\mathrm{osc}}$.
}

After the oscillations begins, the amplitude of the radial direction of the inflaton $S$ decreases with time as $|S| \propto a^{-3 / 2}$. That of the AD field does as $|\phi| \propto a^{-3 / 4}$ so that its number density $\left(H(t)|\phi|^{2} / 2\right)$ decreases as $\propto a^{-3}$. Since the A-term, i.e., the $B-L$ number violating interaction, is a higher dimensional term, it is turned off soon after the AD field starts to oscillate after inflation. The generated $B-L$ asymmetry is then conserved in a comoving volume and thus $n_{B-L} \propto a^{-3}$ for $t>t_{\mathrm{osc}}$.

In the above discussion, we assume that we can take a time average over the period of an inflaton-oscillation time scale. In order to justify the above results, we have numerically solved the equations of motion together with the Friedmann equation, where the waterfall fields are collectively described by a real scalar field $\tilde{\psi}$ such as $\psi=\bar{\psi} \equiv \tilde{\psi} / 2$. We assume $|S|^{2} / M_{\mathrm{Pl}}^{2},|\phi|^{2} / M_{\mathrm{Pl}}^{2}, \tilde{\psi}^{2} / M_{\mathrm{Pl}}^{2} \ll$ 1 and take into account next-to-leading order terms in terms of them (see Appendix). We use the full kinetic terms for $S$ and $\phi$ [see Eq. (13)], while we assume a canonical one for $\psi$ for simplicity. One of the results is shown in Fig. 3, where the generated $B-L$ asymmetry is consistent with Eq. (84). Taking parameters such as $n=4,6$, $\kappa=0.02-0.5, \quad \mu=0.0004-0.02, \quad \lambda=0.01-100, \quad$ and $\theta_{\phi}^{\text {ini }}=0.001-0.1, \quad$ we confirm the above parameter dependences and obtain the numerical uncertainty of $(0.1-0.2)$ in Eq. (85). We assume $c_{H}=-1$ and $c_{2}=0$ in our calculations, but we check that nonzero values of $c_{2}$ $[=\mathcal{O}(1)$ and $\geq 0]$ does not change our results even quantitatively.

\section{B. Baryon asymmetry}

The AD field starts to oscillate just after inflation and generates $B-L$ asymmetry. The oscillating $\mathrm{AD}$ field decays and dissipates into radiation [53] and the sphaleron effect relates the $B-L$ asymmetry to the baryon asymmetry $[3,4]$. Using Eq. (84), we can calculate the baryon-toentropy ratio $Y_{b}$ such as

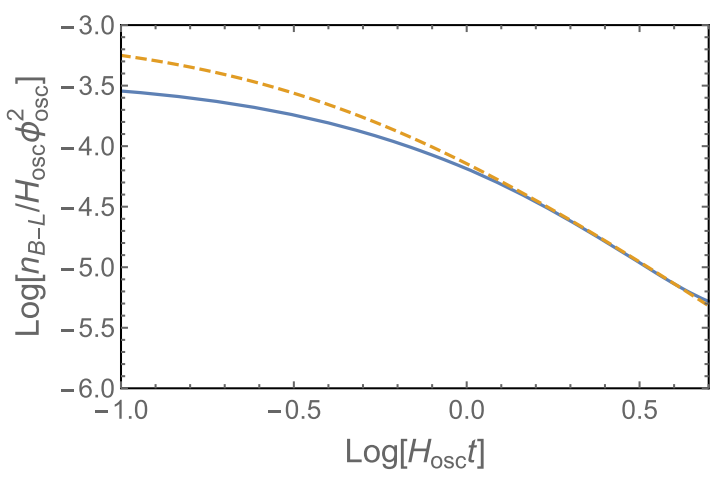

FIG. 3. Evolution plot for $B-L$ number after hybrid inflation. The dashed curve is our prediction of Eq. (85) with a numerical factor of 0.2 . We assume $\lambda=1, n=6, c_{H}=-1, c_{2}=0$, $\kappa=0.05, \mu=0.001$, and $\theta_{\phi}^{\text {ini }}=0.01$. 


$$
\begin{aligned}
Y_{b} & \simeq \frac{8}{23} \frac{\epsilon q T_{\mathrm{RH}}}{4 H_{\mathrm{osc}}}\left(\frac{\phi_{\mathrm{osc}}}{M_{\mathrm{Pl}}}\right)^{2} \\
& \simeq\left\{\begin{array}{l}
0.05 \sqrt{\left|c_{H}\right|} q \frac{\epsilon}{\lambda} \frac{T_{\mathrm{RH}}}{M_{\mathrm{Pl}}} \text { for } n=4 \\
0.06\left|c_{H}\right|^{1 / 4} q \frac{\epsilon}{\lambda^{1 / 2}} \frac{T_{\mathrm{RH}}}{\sqrt{H_{\mathrm{osc}} M_{\mathrm{Pl}}}} \quad \text { for } n=6 .
\end{array}\right.
\end{aligned}
$$

Since $\epsilon \equiv \tilde{\epsilon} S_{\mathrm{cr}} / M_{\mathrm{Pl}}, S_{\mathrm{cr}}=\mu$, and $H_{\mathrm{osc}}^{2} \simeq \kappa^{2} \mu^{4} /\left(3 M_{\mathrm{Pl}}^{2}\right)$, this is rewritten as

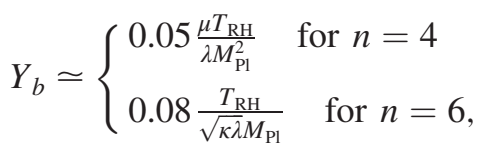

where we assume $\left|c_{H}\right|=1, q=1$, and $\tilde{\epsilon}=1$. For typical parameters, it is given by

$Y_{b} \simeq \begin{cases}9 \times 10^{-11}\left(\frac{\mu}{10^{15} \mathrm{GeV}}\right)\left(\frac{T_{\mathrm{RH}}}{10^{9} \mathrm{GeV}}\right)\left(\frac{\lambda}{10^{-4}}\right)^{-1} & \text { for } n=4 \\ 1 \times 10^{-10} \lambda^{-1 / 2}\left(\frac{\kappa}{10^{-3}}\right)^{-1 / 2}\left(\frac{T_{\mathrm{RH}}}{10^{7} \mathrm{GeV}}\right) & \text { for } n=6 .\end{cases}$

We check that the constraints of Eqs. (75) and (76) and $\xi \leq 1$ [see Eq. (71)] are satisfied for the above reference parameters. Thus, we can explain the observed baryon asymmetry of $Y_{b}^{\text {obs }} \simeq 8.7 \times 10^{-11}$ [55] in this scenario.

Since a linear combination of phase directions is massless during inflation, our scenario predicts nonzero baryonic isocurvature fluctuations like the case in Sec. II D. However, the energy scale of hybrid inflation can be lower than the constraint of Eq. (54). In fact, for the above reference parameters, our scenario is consistent with the present upper bound on the isocurvature mode.

\section{Reheating temperature}

As we can see in Eq. (89), the resulting baryon asymmetry depends on reheating temperature $T_{\mathrm{RH}}$. To determine it, let us consider the decay of inflaton. There is a lower bound on the reheating temperature because the inflaton decays into the MSSM particles via supergravity effects. The decay rate is calculated as

$$
\Gamma_{\mathrm{inf}}^{\mathrm{SUGRA}}=\frac{3}{128 \pi^{3}}\left|y_{t}\right|^{2}\left(\frac{\mu}{M_{\mathrm{Pl}}}\right)^{2} \frac{m_{\mathrm{inf}}^{3}}{M_{\mathrm{Pl}}^{2}},
$$

where $m_{\text {inf }}=\sqrt{2} \kappa \mu$ is the inflaton mass and $y_{t}$ is the top Yukawa coupling constant. The lower bound on the reheating temperature is thus given by [64]

$T_{\mathrm{RH}}^{(\min )} \simeq 3 \times 10^{3} \mathrm{GeV}\left|y_{t}\right|\left(\frac{\mu}{10^{15} \mathrm{GeV}}\right)\left(\frac{m_{\mathrm{inf}}}{10^{12} \mathrm{GeV}}\right)^{3 / 2}$.
If there is an interaction between the inflaton and Higgs fields such as

$$
W \supset y \phi H_{u} H_{d},
$$

then the inflaton decay rate and the reheating temperature are estimated as

$$
\begin{aligned}
\Gamma_{\mathrm{inf}} & =\frac{y^{2}}{4 \pi} m_{\phi} \\
T_{\mathrm{RH}} & \simeq 2 \times 10^{10} \mathrm{GeV}\left(\frac{y}{10^{-4}}\right)\left(\frac{m_{\mathrm{inf}}}{10^{12} \mathrm{GeV}}\right)^{1 / 2} .
\end{aligned}
$$

Note that the coupling constant $y$ should be smaller than $\kappa$ so as not to affect the Coleman-Weinberg potential of Eq. (64). Thus the reheating temperature cannot be higher than that of Eq. (94) with $y \approx \kappa$.

We have to take into account the constraint on $T_{\mathrm{RH}}$ from gravitino overproduction problems. The inflaton decays also into gravitinos via supergravity effects. Its production rate is given by [64]

$$
\Gamma_{3 / 2} \simeq \frac{1}{96 \pi}\left(\frac{\mu}{M_{\mathrm{Pl}}}\right)^{2} \frac{m_{\mathrm{inf}}^{3}}{M_{\mathrm{Pl}}^{2}}
$$

The resulting gravitino-to-entropy ratio from this contribution is given by

$$
Y_{3 / 2}^{(\text {decay })} \simeq \frac{3}{2}\left(\frac{90}{g_{*} \pi^{2}}\right)^{1 / 2} \frac{\Gamma_{3 / 2} M_{\mathrm{Pl}}}{m_{\mathrm{inf}} T_{\mathrm{RH}}}
$$

Gravitinos are also produced from scatterings in the thermal plasma after reheating completes. Its abundance is given by [65-67]

$$
\begin{aligned}
Y_{3 / 2}^{(\text {thermal })} \simeq & 0.26 \frac{\rho_{c}}{m_{3 / 2} s_{0}}\left(\frac{T_{\mathrm{RH}}}{10^{10} \mathrm{GeV}}\right)\left[0.13\left(\frac{m_{3 / 2}}{100 \mathrm{GeV}}\right)\right. \\
& \left.+\left(\frac{100 \mathrm{GeV}}{m_{3 / 2}}\right)\left(\frac{m_{\tilde{g}}}{1 \mathrm{TeV}}\right)^{2}\right]
\end{aligned}
$$

where $s_{0} \quad\left(\simeq 2.9 \times 10^{3} \mathrm{~cm}^{-3}\right) \quad$ and $\rho_{c} \quad(\simeq 1.052 \times$ $\left.10^{-5} h^{2} \mathrm{GeV} / \mathrm{cm}^{3}\right)$ are the present entropy density and critical energy density, respectively. The parameter $m_{\tilde{g}}$ is gluino mass and $h$ is the present Hubble parameter in the unit of $100 \mathrm{~km} \mathrm{~s}^{-1} \mathrm{Mpc}^{-1}$. Stringent bounds on the reheating temperature are obtained when we assume that the gravitino is the lightest SUSY particle (LSP) and is stable. In this case, its abundance should not exceed the observed DM abundance:

$$
m_{3 / 2}\left(Y_{3 / 2}^{(\text {decay })}+Y_{3 / 2}^{(\text {thermal })}\right) \leq \frac{\rho_{c}}{s_{0}} \Omega_{\mathrm{DM}} \simeq 0.4 \mathrm{eV}
$$


where $\Omega_{\mathrm{DM}} h^{2}(\simeq 0.12)$ is the DM relic density. ${ }^{11}$ For example, in the case of $m_{3 / 2}=100 \mathrm{GeV}$, the reheating temperature is bounded such as

$$
\begin{aligned}
2 \times & 10^{7} \mathrm{GeV}\left(\frac{\mu}{10^{15} \mathrm{GeV}}\right)^{2}\left(\frac{m_{\mathrm{inf}}}{10^{12} \mathrm{GeV}}\right)^{2} \\
& \lesssim T_{\mathrm{RH}} \lesssim 9 \times 10^{9} \mathrm{GeV},
\end{aligned}
$$

where we use $h \simeq 0.67$. We can see that the reference parameters used in Eq. (89) are consistent with this bound.

Note that for the case of $n=4$, the coupling constant in the superpotential of the AD field cannot be much larger than $10^{-4}$ because of the upper bound on the reheating temperature. For the case of $n=6$, we can naturally explain the observed baryon asymmetry for $\lambda=\mathcal{O}(1)$ with a reheating temperature consistent with the gravitino problem. This is in contrast to the result in the conventional scenario of ADBG [see Eq. (36)], where an extremely large value of $\lambda$ is required to be consistent with the lower bound on the reheating temperature. In the case of such a large value of $\lambda$, the thermal $\log$ potential has to be taken into account even for $n=6$.

\section{CHAOTIC INFLATION}

In this section, we consider our scenario of ADBG in a chaotic inflation model with a shift symmetry in supergravity $[69,70]$. The inflaton $I$ has a shift symmetry in the Kähler potential and the minimal Kähler potential is written as

$K_{\mathrm{inf}}=c_{0} M_{\mathrm{Pl}}\left(I+I^{*}\right)+\frac{1}{2}\left(I+I^{*}\right)^{2}+|X|^{2}-\frac{c_{3}}{4} \frac{|X|^{4}}{M_{\mathrm{Pl}}^{2}}$,

where $X$ is a stabilizer field. Note that $c_{0}$ is an order parameter of $Z_{2}$ symmetry, under which the fields $I$ and $X$ are odd, so that we take $c_{0}$ as a free parameter that may be smaller than unity. We include the $|X|^{4}$ term in the Kähler potential, which cannot be suppressed by any symmetries. The other higher dimensional terms do not change our discussion qualitatively, so that we neglect them in the following analysis.

\footnotetext{
${ }^{11}$ If the gravitino mass is about $1 \mathrm{TeV}$ and it is unstable, its decay products interact with the light elements and destroy them at the time of BBN epoch. Then the gravitino abundance is bounded above by about four order of magnitude more severe than the bound of Eq. (98) [68]. On the other hand, if the gravitino is stable, the next to LSP (NLSP) decays at the time of BBN epoch and its decay products may destroy light elements. This constraint is severe for bino or stau NLSP, while it can be easily avoided for sneutrino NLSP [68]. In this paper we use the conservative bound of Eq. (98), which is independent of lowenergy SUSY models.
}

To realize chaotic inflation in a quadratic potential, the superpotential is assumed to break the shift symmetry such as

$$
W_{\mathrm{inf}}=m_{\mathrm{inf}} I X,
$$

where $m_{\text {inf }}$ is inflaton mass. The field $I$ has a quadratic potential from the F-term of $X$. Its imaginary component can have a larger VEV than the Planck scale thanks to the shift symmetry in the Kähler potential and is identified with the inflaton. The real component of $I$ obtains a Hubbleinduced mass and stays at a VEV of $\operatorname{Re}[I]=-c_{0} / 2$ [71]. When the VEV of the inflaton decreases down to the Planck scale, the real component of $I$ as well as the inflaton start to oscillate around the origin of the potential and inflation ends. The dynamics is illustrated in Fig. 4, where we numerically solve the equation of motion of the field $I$ and plot its trajectory for the case of $c_{0}=1$. The field $I$ slowly rolls along the imaginary axis during inflation, where $\operatorname{Re}[I]=-c_{0} / 2$ is approximately satisfied. After it reaches the red point, inflation ends and it starts to oscillate and rotate around the origin. The Hubble parameter at the end of inflation is given by

$$
H_{\mathrm{osc}} \simeq \frac{m_{\mathrm{inf}}}{\sqrt{3}} .
$$

The stabilizer field $X$ obtains a Hubble-induced mass via the higher dimensional Kähler potential such as

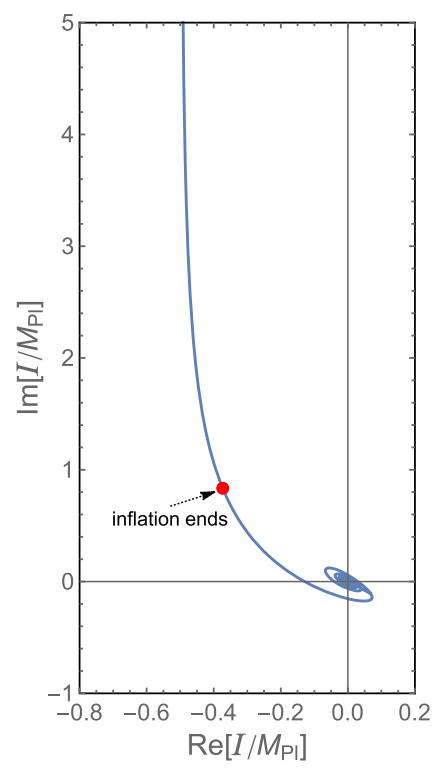

FIG. 4. Dynamics of the field $I$ in the complex plane in the chaotic inflation model. We set $c_{0}=1$. The field $I$ slowly rolls along the line of $\operatorname{Re}[I]=-c_{0} / 2$ during inflation. After it reaches the red point, inflation ends and it starts to oscillate and rotate around the origin. 


$$
V \supset c_{3} m^{2} \frac{|I|^{2}}{M_{\mathrm{Pl}}^{2}}|X|^{2} \simeq 3 c_{3} H^{2}|X|^{2} .
$$

This implies that the dynamics of $X$ is qualitatively different from the case with $c_{3}=0$. We should include them because the higher dimensional Kähler potential cannot be suppressed by any symmetries. To realize chaotic inflation, we assume $c_{3}>0$. Then the field $X$ stays at the origin. However, when we take into account of the backreaction of the $\mathrm{AD}$ field, $X$ obtains a small VEV as shown in the next subsection.

\section{A. Dynamics of the AD field}

Taking into account the AD field, we consider the Kähler potential of

$K=K_{\mathrm{inf}}+|\phi|^{2}+c_{1}|X|^{2}|\phi|^{2}-\frac{c_{2}}{2}\left(I+I^{*}\right)^{2}|\phi|^{2}$.

Although we introduce a shift symmetry for the field $I$, the fields $X$ and $I$ basically correspond to the fields $S$ and $\psi$ in Eq. (55), respectively. The AD field acquires the Hubbleinduced mass term from the F-term of $X$ during inflation. After inflation ends, the Hubble-induced mass term partially comes from kinetic interactions. In fact, the Kähler potential of $-c_{2} / 2\left(I+I^{*}\right)^{2}|\phi|^{2}$ induces a kinetic interaction of

$$
\mathcal{L} \supset-c_{2} \frac{1}{M_{\mathrm{Pl}}^{2}}|\phi|^{2}\left|\partial_{\mu} I\right|^{2}
$$

We obtain the effective Hubble-induced mass term of $\left(3 c_{2} / 2\right) H^{2}(t)|\phi|^{2}$ from this kinetic interaction. To sum up, the Hubble-induced mass term is given by

$$
\begin{aligned}
V_{H} & =c_{H} H^{2}(t)|\phi|^{2} \\
c_{H} & = \begin{cases}-3\left(c_{1}-1\right) & \text { during inflation } \\
\frac{3}{2}\left(c_{2}-c_{1}+1\right) & \text { after inflation, }\end{cases}
\end{aligned}
$$

where the other terms than the one proportional to $c_{2}$ come from the potential energy. Thus we can consider the case that the coefficient $c_{H}$ is negative during inflation and is positive after inflation.

There is also an A-term such as

$$
\begin{aligned}
V_{A} & =\frac{1}{n}\left(n\left(1-c_{1}\right)-2\right) \frac{\lambda m_{\mathrm{inf}}}{M_{\mathrm{Pl}}^{n-1}} I X\left(\phi^{*}\right)^{n}+\text { c.c. } \\
& =\frac{2}{n}\left(n\left(1-c_{1}\right)-2\right) \frac{\lambda m_{\mathrm{inf}}}{M_{\mathrm{Pl}}^{n-1}}|I||X||\phi|^{n} \cos \left(\theta_{I}+\theta_{X}-n \theta_{\phi}\right) \\
& \simeq-a H^{2}(t) \frac{|X|}{M_{\mathrm{Pl}}}|\phi|^{2} \cos \left(\theta_{I}+\theta_{X}-n \theta_{\phi}\right)
\end{aligned}
$$

where we use Eq. (25) and $H(t) \simeq m_{\text {inf }}|I| / \sqrt{3} M_{\mathrm{Pl}}$ in the last line and $\theta_{I}, \theta_{X}$, and $\theta_{\phi}$ are the complex phases of the fields $I, X$, and $\phi$, respectively. The coefficient $a$ is given by

$$
a=2 \sqrt{\frac{3\left|c_{H}\right|}{n-1}}\left(c_{1}-1+\frac{2}{n}\right) .
$$

The A-term can be regarded as a linear term for $X$. Since the field $X$ has a positive Hubble-induced mass term of Eq. (103), it stays at the following minimum during inflation:

$$
\langle|X|\rangle \simeq \frac{a}{6 c_{3}} \frac{1}{M_{\mathrm{Pl}}}|\phi|^{2} .
$$

A linear combination of the phase directions has a mass of order the Hubble parameter due to the A-term, so that it stays at the following minimum during inflation:

$$
\left\langle\theta_{X}-n \theta_{\phi}\right\rangle=-\left\langle\theta_{I}\right\rangle \simeq-\operatorname{sign}\left[c_{0}\right] \frac{\pi}{2},
$$

where we use $\operatorname{Re}[I] \ll \operatorname{Im}[I]$ during inflation.

After inflation ends, the field $I$ starts to rotate in the phase space as shown in Fig. 1 and its phase $\theta_{I}$ has a nonzero velocity. This implies that a linear combination of the phases $\theta_{X}$ and $\theta_{\phi}$ obtains a nonzero velocity to follow its potential minimum. Since the A-term contains the phase direction of the inflaton, the whole dynamics is difficult to imagine. In fact, one may estimate $\epsilon \approx a|X|_{\text {osc }} / M_{\mathrm{Pl}}$ like the case in the hybrid inflation model considered in the previous section [see Eq. (84)], but we find this estimation wrong. We perform numerical calculations to solve the equations of motion for the complex scalar fields $S, X$, and $\phi$. We use the full supergravity potential for $S, X$, and $\phi$. The kinetic interactions are simplified such that $S$ and $X$ have canonical kinetic terms for simplicity. We take into account the kinetic interactions for $\phi$ associated with $c_{2}$, which is needed to change the sign of its Hubble-induced mass term. The parameters are taken in the intervals of $\lambda=10^{-3}-10^{4}$ and $c_{0}=10^{-5}-1$ for $n=4$ and 6 . The $\mathcal{O}(1)$ coefficients in the Kähler potential are assumed to be $c_{1}=2, c_{2}=1$, and $c_{3}=1$. From our numerical calculations, we obtain the following results:

$$
\begin{aligned}
\frac{a^{3}(t)}{a^{3}\left(t_{\mathrm{osc}}\right)} n_{B-L}(t) & \equiv \epsilon q H_{\mathrm{osc}} \phi_{\mathrm{osc}}^{2} \\
\epsilon & \equiv \tilde{\epsilon} c_{0} \\
\tilde{\epsilon} & \simeq(0.01-0.1) a,
\end{aligned}
$$

where the factor of $0.01-0.1$ is a numerical uncertainty. One example of our results is shown in Fig. 5, where we set $\lambda=1, n=6, c_{0}=0.5, c_{1}=2,\left|c_{2}\right|=1$, and $c_{3}=-1$. The 


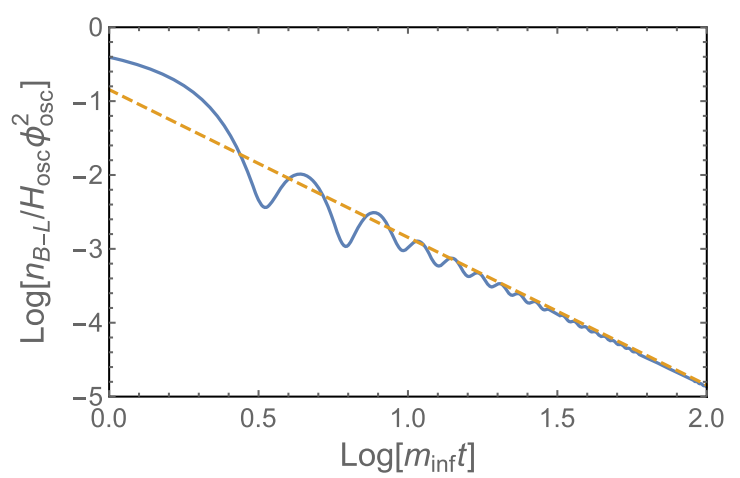

FIG. 5. Evolution plot for $B-L$ number density in our scenario of ADBG in the chaotic inflation model. The dashed curve is our prediction of Eq. (116) with a numerical factor of 0.01. We take $\lambda=1, n=6, c_{0}=0.5, c_{1}=2,\left|c_{2}\right|=1$, and $c_{3}=-1$.

blue curve represents the time evolution of the $B-L$ number after the end of inflation, while the orange dashed curve corresponds to Eq. (116) with a numerical factor of 0.01 . The oscillation behavior of $B-L$ number density may come from the effect of the oscillating inflaton through supergravity effects and is irrelevant for our discussion. ${ }^{12}$ The $c_{0}$ dependence in our result of Eq. (115) comes from the ellipticity of the dynamics of the inflaton in the complex plane. This means that $B-L$ asymmetry cannot be generated for $c_{0}=0$, in which case no $C P$ odd component of the field $I$ is excited.

One might wonder why there is no factor of $|X|$ in our result of Eq. (115) in contrast to the one in the case of hybrid inflation [see Eq. (84)]. Although we perform numerical calculation with the full supergravity potential with some kinetic interactions to derive the above results, we also check the same parameter dependence in the following toy model:

$$
\begin{array}{r}
\ddot{\phi}+3 H(t) \dot{\phi}+H^{2}(t) \phi-n a_{H} I X\left(\phi^{*}\right)^{n-1}=0, \\
\ddot{I}+3 H(t) \dot{I}+m^{2} I-a_{H} X^{*} \phi^{n}=0, \\
\ddot{X}+3 H(t) \dot{X}+m^{2} X-a_{H} I^{*} \phi^{n}=0,
\end{array}
$$

where $H(t)=2 / 3 t$. Initial conditions are taken as

$$
\begin{aligned}
& \phi\left(t_{0}\right)=1, \quad I\left(t_{0}\right)=1, \quad X\left(t_{0}\right)=X_{0}, \\
& \dot{\phi}\left(t_{0}\right)=0, \quad \dot{I}\left(t_{0}\right)=i c_{0}, \quad \dot{X}\left(t_{0}\right)=0 .
\end{aligned}
$$

\footnotetext{
${ }^{12}$ We have investigated a possibility to generate $B-L$ asymmetry via this effect in Ref. [72]. Note that in this paper we do not introduce a $B-L$ violating operator associated with the righthanded neutrino, so that the net $B-L$ asymmetry vanishes for this effect. Even if we introduce the $B-L$ violating operator, the resulting $B-L$ asymmetry generated from this effect is much smaller than that generated from ADBG.
}

We confirm that the resulting $B-L$ density is proportional to $c_{0}$ and $a_{H}$, and is almost independent of $X_{0}$.

\section{B. Baryon asymmetry}

Using the results obtained in the previous subsection, we calculate the baryon-to-entropy ratio such as

$$
\begin{aligned}
Y_{b} & \simeq \frac{2 \tilde{\epsilon} q}{23} c_{0} \frac{T_{\mathrm{RH}}}{H_{\mathrm{osc}}}\left(\frac{\phi_{\mathrm{osc}}}{M_{\mathrm{Pl}}}\right)^{2} \\
& \simeq \begin{cases}0.005 c_{0} \frac{T_{\mathrm{RH}}}{\lambda M_{\mathrm{Pl}}} & \text { for } n=4 \\
0.006 c_{0} \frac{T_{\mathrm{RH}}}{\sqrt{\lambda H_{\mathrm{osc}} M_{\mathrm{Pl}}}} & \text { for } n=6,\end{cases}
\end{aligned}
$$

where we assume $\tilde{\epsilon} q=0.1$ and $\left|c_{H}\right|=1$ in the last line. For typical parameters, it is given by

$Y_{b} \simeq\left\{\begin{array}{l}2 \times 10^{-10}\left(\frac{c_{0} T_{\mathrm{RH}}}{10^{7} \mathrm{GeV}}\right)\left(\frac{\lambda}{10^{-4}}\right)^{-1} \text { for } n=4 \\ 1 \times 10^{-10}\left(\frac{c_{0} T_{\mathrm{RH}}}{10^{6} \mathrm{GeV}}\right)\left(\frac{\lambda}{10^{-4}}\right)^{-1 / 2} \text { for } n=6,\end{array}\right.$

where we use $H_{\mathrm{osc}} \simeq m_{\mathrm{inf}} \approx 10^{13} \mathrm{GeV}$. Thus, we can explain the observed baryon asymmetry of $Y_{b}^{\text {obs }} \simeq 8.7 \times 10^{-11}$ [55].

Since the COBE normalization of the amplitude of density perturbations requires that the energy scale of chaotic inflation is given by $H_{\text {inf }} \simeq 10^{14} \mathrm{GeV}$ in the chaotic inflation model, the baryonic isocurvature constraint of Eq. (54) is much more severe than the case in the hybrid inflation. It requires that the parameter in the superpotential $\lambda$ is smaller than about $10^{-4}$. This means that the VEV of the $\mathrm{AD}$ field is as large as the Planck scale during inflation. In this case, the backreaction of the $\mathrm{AD}$ field to inflaton dynamics might be relevant. As a result, the tensor-to-scalar ratio can be consistent with the present constraint within $2 \sigma[11,73]$. Note that the number density of the AD field decreases with time as $\propto a^{-3}$ due to the expansion of the Universe. This means that its energy density decreases as $a^{-9 / 2}$ because its effective mass is of order the Hubble parameter, which decreases as $a^{-3 / 2}$. Thus its energy density never dominates that of the Universe and the result of Eq. (122) is applicable even for the case of $\phi_{\mathrm{osc}} \simeq M_{\mathrm{Pl}}$.

\section{Reheating temperature}

The inflaton can decay into the MSSM particles via supergravity effects. Its decay rate is calculated in Ref. [64] and is given as

$$
\Gamma_{\mathrm{inf}}^{(\mathrm{SUGRA})}=\frac{3 c_{0}^{2}}{256 \pi^{3}}\left|y_{t}\right|^{2} \frac{m_{\mathrm{inf}}^{3}}{M_{\mathrm{Pl}}^{2}} .
$$

This implies that the reheating temperature is given by 


$$
T_{\mathrm{RH}} \simeq 2 \times 10^{8} \mathrm{GeV} c_{0}\left|y_{t}\right|\left(\frac{m_{\mathrm{inf}}}{10^{13} \mathrm{GeV}}\right)^{3 / 2} .
$$

Together with Eq. (124), we find that the observed baryon asymmetry can be explained when $c_{0}=\mathcal{O}(0.1)$.

Note that there may be a renormalizable coupling such as

$$
W \supset y X H_{u} H_{d} .
$$

If $c_{0}$ is sufficiently small, the decay rate is determined by this term and is given by

$$
T_{\mathrm{RH}} \simeq 6 \times 10^{8} \mathrm{GeV}\left(\frac{y}{10^{-6}}\right)\left(\frac{m_{\mathrm{inf}}}{10^{13} \mathrm{GeV}}\right)^{1 / 2} .
$$

However, the coupling constant $y$ should be suppressed by a factor of $m_{\mathrm{inf}} / M_{\mathrm{Pl}}$ not to affect the inflaton potential, so that the reheating temperature is at most $10^{9} \mathrm{GeV}$ [74].

In order to kick the phase direction and generate $B-L$ asymmetry, we need a nonzero value of $Z_{2}$ breaking parameter $c_{0}$. However, the $Z_{2}$ breaking term makes the inflaton decay into gravitinos efficiently via supergravity effects and its decay rate is the same order with that of Eq. (125). Therefore, there is a gravitino problem from inflaton decay. We can avoid the problem by assuming that the gravitino is sufficiently heavy $\left(m_{3 / 2} \gtrsim 100 \mathrm{TeV}\right)$ so as to decay before the BBN epoch and the R-parity is violated for the LSP not to overclose the Universe. Or, we can assume that gravitino is sufficiently light $\left(m_{3 / 2} \lesssim 2 \mathrm{keV}\right)$, in which case they do not overclose the Universe. The former possibility might be well motivated partly because the observed $125 \mathrm{GeV}$ Higgs mass favors a heavy squark mass of order $100 \mathrm{TeV}$ for a small $\tan \beta$ [75-78].

\section{DISCUSSION AND CONCLUSIONS}

We have investigated a new scenario that the AffleckDine mechanism works just after the end of inflation. The AD field stays at a large VEV by a negative Hubbleinduced mass term during inflation and then starts to oscillate around the origin by a positive one after inflation. At the same time, its phase direction is kicked by an A-term and $B-L$ asymmetry is generated. Since its dynamics is determined by Hubble-induced terms, the resulting $B-L$ asymmetry is independent of parameters in low-energy SUSY models. This fact makes our scenario very simple. In particular, Q-balls, which sometimes form after the conventional scenario of ADBG, do not form in our scenario.
The A-term depends on inflation models, so that the resulting $B-L$ asymmetry does in our scenario. We have investigated the scenario and calculated the produced amount of $B-L$ asymmetry in F-term hybrid and chaotic inflation models in supergravity. We have found that our scenario requires a higher reheating temperature than the one required in the conventional scenario. This implies that ADBG works in larger parameter spaces than expected in the literature. In particular, in the F-term hybrid inflation model, the required reheating temperature is naturally consistent with the gravitino overproduction bounds.

The required reheating temperature is not unnaturally small even if the VEV of the AD field is so large that its backreaction to inflaton dynamics becomes relevant. Since the backreaction can make the spectral index and tensor-toscalar ratio consistent with observations [11,73], this is another advantage of this scenario.

\section{ACKNOWLEDGMENTS}

M. Y. thanks W. Buchmüller for kind hospitality at DESY. This work is supported by World Premier International Research Center Initiative (WPI Initiative), MEXT, Japan, the Program for the Leading Graduate Schools, MEXT, Japan, and the JSPS Research Fellowships for Young Scientists, No. 25.8715.

\section{APPENDIX: EQUATIONS OF MOTION}

In this appendix, we write the equations of motion for the $\mathrm{AD}$ field and fields in inflaton sectors, which we use in numerical simulations.

In the case of hybrid inflation, we solve the following equations of motion of two complex scalar fields and one real scalar field, $\phi, S$, and $\tilde{\psi}$, together with the Friedmann equation which determines the evolution of the Hubble parameter $H(t)$, where we collectively describe the waterfall fields by a real scalar field $\tilde{\psi}$ such as $\psi=\bar{\psi}=\tilde{\psi} / 2$. We assume $|S|^{2} / M_{\mathrm{Pl}}^{2},|\phi|^{2} / M_{\mathrm{Pl}}^{2}, \tilde{\psi}^{2} / M_{\mathrm{Pl}}^{2} \ll 1$ and take into account next-to-leading order terms in terms of them in Eq. (7). Including the 1-loop Coleman-Weinberg potential, we write the potential such as

$$
V=V_{U(1)}+V_{A}+V_{\mathrm{CW}}
$$

where $V_{A}$ and $V_{\mathrm{CW}}$ are given by Eqs. (68) and (64), respectively, and $V_{U(1)}$ is given by

$$
\begin{aligned}
V_{U(1)} \simeq & {\left[1+\frac{\tilde{\psi}^{2}}{2 M_{\mathrm{Pl}}^{2}}+\left(1-c_{1}\right) \frac{|\phi|^{2}}{M_{\mathrm{Pl}}^{2}}+\left(1-c_{1}\right) \frac{|\phi|^{2}|S|^{2}}{M_{\mathrm{Pl}}^{4}}+\frac{|S|^{4}}{2 M_{\mathrm{Pl}}^{4}}\right]\left|\kappa\left(\frac{\tilde{\psi}^{2}}{4}-\mu^{2}\right)\right|^{2} } \\
& +\frac{1}{2} \kappa^{2}|S|^{2} \tilde{\psi}^{2}\left(1+\frac{|\phi|^{2}}{M_{\mathrm{Pl}}^{2}}\right)+\left[1+\left(1-c_{1}\right) \frac{|S|^{2}}{M_{\mathrm{Pl}}^{2}}\right] \lambda^{2} \frac{|\phi|^{2 n-2}}{M_{\mathrm{Pl}}^{2 n-6}}
\end{aligned}
$$


where we have assumed $c_{2}=0$. In our numerical simulation, we set $c_{1}=4 / 3$ (i.e., $c_{H}=-1$ ). Since we consider the nonminimal Kähler potential given as Eq. (55), the kinetic term is not canonical [see Eq. (13)]. Therefore, the equations of motion of scalar fields are written as

$a^{-3} \frac{\mathrm{d}}{\mathrm{d} t}\left[a^{3} K_{i \bar{j}} \frac{\mathrm{d}}{\mathrm{d} t} \phi^{i}\right]-K_{i \bar{l} \bar{j}}\left(\frac{\mathrm{d}}{\mathrm{d} t} \phi^{i}\right)\left(\frac{\mathrm{d}}{\mathrm{d} t} \phi^{* \bar{l}}\right)+V_{\bar{j}}=0$,

where the subscripts represent the derivatives with respect to corresponding fields, e.g., $K_{i \bar{j}}=\partial^{2} K / \partial \phi \partial S^{*}$ for $i=\phi$ and $\bar{j}=\bar{S}$. This is rewritten as

$K_{i \bar{j}} \ddot{\phi}^{i}+\dot{K}_{i \bar{j}} \dot{\phi}^{i}+3 H(t) K_{i \bar{j}} \dot{\phi}^{i}-K_{i \bar{l} \bar{j}} \dot{\phi}^{i} \dot{\phi}^{* \bar{l}}+V_{\bar{j}}=0$,

where $K$ is given by Eq. (55). The Friedmann equation is written as

$H^{2}(t) \simeq \frac{1}{3 M_{\mathrm{Pl}}^{2}}\left[V_{U(1)}+V_{\mathrm{CW}}+(\right.$ Kinetic energy $\left.)\right]$

where we neglect $V_{A}$ for simplicity. We can approximate the kinetic energy as

$$
(\text { Kinetic energy }) \simeq|\dot{\phi}|^{2}+|\dot{S}|^{2}+\frac{1}{2} \dot{\tilde{\psi}}^{2},
$$

because higher-dimensional terms are much smaller than these terms and are irrelevant.

In the case of chaotic inflation, we solve the following equations of motion of three complex scalar fields, $\phi, S$, and $X$, together with the Friedmann equation which determines the evolution of the Hubble parameter $H(t)$. We use the full supergravity potential of Eq. (7), where $W$ is given by the sum of Eqs. (17) and (101) and $K$ is given by Eq. (104). We neglect the effects of nonminimal kinetic terms on $S$ and $X$ because the VEV of the AD field is much smaller than the Planck scale. Thus we write their equation of motion as

$$
\begin{aligned}
& \ddot{S}+3 H(t) \dot{S}+\frac{\partial}{\partial S^{*}} V_{\text {SUGRA }}=0, \\
& \ddot{X}+3 H(t) \dot{X}+\frac{\partial}{\partial X^{*}} V_{\text {SUGRA }}=0 .
\end{aligned}
$$

We take into account some nonminimal kinetic interactions for $\phi$ that are relevant to realize our scenario of ADBG:

$\tilde{K}_{i \bar{j}} \ddot{\phi}^{i}+\dot{\tilde{K}}_{i \bar{j}} \dot{\phi}^{i}+3 H(t) \tilde{K}_{i \bar{j}} \dot{\phi}^{i}-\tilde{K}_{i \bar{l} \bar{j}} \dot{\phi}^{i} \dot{\phi}^{* \bar{l}}+\frac{\partial}{\partial \phi^{*}} V_{\text {SUGRA }}=0$,

where $j=\phi$ and

$$
\tilde{K}=|\phi|^{2}-\frac{c_{2}}{2 M_{\mathrm{Pl}}^{2}}\left(I+I^{*}\right)^{2}|\phi|^{2} .
$$

The nonminimal kinetic terms associated with $c_{2}$ make $\phi$ have a positive Hubble-induced mass after inflation [see Eq. (105)], while the other terms, which we neglect in our numerical calculations, are irrelevant for our discussion due to the smallness of the VEV of $X$ and $\phi$. The Friedmann equation is given by Eq. (A5), where we can use canonical kinetic terms for $\phi, S$, and $X$.
[1] I. Affleck and M. Dine, Nucl. Phys. B249, 361 (1985).

[2] M. Dine, L. Randall, and S. D. Thomas, Nucl. Phys. B458, 291 (1996).

[3] V. A. Kuzmin, V. A. Rubakov, and M. E. Shaposhnikov, Phys. Lett. 155B, 36 (1985).

[4] M. Fukugita and T. Yanagida, Phys. Lett. B 174, 45 (1986).

[5] A. Anisimov and M. Dine, Nucl. Phys. B619, 729 (2001).

[6] M. Fujii, K. Hamaguchi, and T. Yanagida, Phys. Rev. D 63, 123513 (2001).

[7] A. Mazumdar and A. Perez-Lorenzana, Phys. Rev. D 65, 107301 (2002).

[8] H. Baer, A. D. Box, and H. Summy, J. High Energy Phys. 08 (2009) 080 (2009).

[9] K. Choi, E. J. Chun, H. D. Kim, W. I. Park, and C. S. Shin, Phys. Rev. D 83, 123503 (2011).
[10] S. Kasuya and M. Kawasaki, Phys. Lett. B 739, 174 (2014).

[11] M. Yamada, Phys. Lett. B 754, 208 (2016).

[12] K. Enqvist and J. McDonald, Phys. Lett. B 425, 309 (1998); Nucl. Phys. B538, 321 (1999).

[13] M. Fujii and K. Hamaguchi, Phys. Lett. B 525, 143 (2002); Phys. Rev. D 66, 083501 (2002).

[14] L. Roszkowski and O. Seto, Phys. Rev. Lett. 98, 161304 (2007).

[15] R. Kitano, H. Murayama, and M. Ratz, Phys. Lett. B 669 , 145 (2008).

[16] I. M. Shoemaker and A. Kusenko, Phys. Rev. D 80, 075021 (2009); S. Kasuya and M. Kawasaki, Phys. Rev. D 84, 123528 (2011); F. Doddato and J. McDonald, J. Cosmol. Astropart. Phys. 07 (2013) 004; S. Kasuya, M. Kawasaki, and M. Yamada, Phys. Lett. B 726, 1 (2013).

[17] G. Kane, J. Shao, S. Watson, and H. B. Yu, J. Cosmol. Astropart. Phys. 11 (2011) 012. 
[18] A. Kamada, M. Kawasaki, and M. Yamada, Phys. Lett. B 719, 9 (2013); Phys. Rev. D 91, 081301 (2015).

[19] K. Harigaya, A. Kamada, M. Kawasaki, K. Mukaida, and M. Yamada, Phys. Rev. D 90, 043510 (2014).

[20] M. Kawasaki and M. Yamada, Phys. Rev. D 91, 083512 (2015).

[21] G. N. Felder, H. Kim, W. I. Park, and E. D. Stewart, J. Cosmol. Astropart. Phys. 06 (2007) 005.

[22] M. Kawasaki and K. Nakayama, Phys. Rev. D 76, 043502 (2007).

[23] K. Choi, K. S. Jeong, W. I. Park, and C. S. Shin, J. Cosmol. Astropart. Phys. 11 (2009) 018.

[24] K. Furuuchi and C. M. Lin, J. Cosmol. Astropart. Phys. 03 (2012) 024.

[25] T. Higaki, K. Kamada, and F. Takahashi, J. High Energy Phys. 09 (2012) 043.

[26] M. A. G. Garcia and K. A. Olive, J. Cosmol. Astropart. Phys. 09 (2013) 007.

[27] T. Hayakawa, M. Kawasaki, and M. Yamada, Phys. Rev. D 93, 063529 (2016).

[28] N. F. Bell, K. Petraki, I. M. Shoemaker, and R. R. Volkas, Phys. Rev. D 84, 123505 (2011).

[29] C. Cheung and K. M. Zurek, Phys. Rev. D 84, 035007 (2011).

[30] W. Fischler, D. Lorshbough, and W. Tangarife, Phys. Rev. D 91, 025010 (2015).

[31] Y. Y. Charng, D. S. Lee, C. N. Leung, and K. W. Ng, Phys. Rev. D 80, 063519 (2009).

[32] M. P. Hertzberg and J. Karouby, Phys. Rev. D 89, 063523 (2014).

[33] M. P. Hertzberg and J. Karouby, Phys. Lett. B 737, 34 (2014).

[34] S. Kasuya and M. Kawasaki, Phys. Rev. D 74, 063507 (2006).

[35] B. Dutta and K. Sinha, Phys. Rev. D 82, 095003 (2010).

[36] D. Marsh, J. High Energy Phys. 05 (2012) 041.

[37] B. Dutta and K. Sinha, Phys. Rev. D 86, 103517 (2012).

[38] J. McDonald, Phys. Lett. B 456, 118 (1999).

[39] A. Kamada and M. Yamada, Phys. Rev. D 91, 063529 (2015).

[40] A. Kamada and M. Yamada, J. Cosmol. Astropart. Phys. 10 (2015) 021.

[41] S. Coleman, Nucl. Phys. B262, 263 (1985).

[42] A. Kusenko, Phys. Lett. B 405 (1997) 108.

[43] A. Kusenko and M. E. Shaposhnikov, Phys. Lett. B 418, 46 (1998).

[44] S. Kasuya and M. Kawasaki, Phys. Rev. D 61, 041301(R) (2000); 62, 023512 (2000); 64, 123515 (2001).

[45] H. Murayama and T. Yanagida, Phys. Lett. B 322, 349 (1994).

[46] T. Gherghetta, C. F. Kolda, and S. P. Martin, Nucl. Phys. B468, 37 (1996).

[47] A. de Gouvea, T. Moroi, and H. Murayama, Phys. Rev. D 56, 1281 (1997).

[48] S. Kasuya, M. Kawasaki, and F. Takahashi, J. Cosmol. Astropart. Phys. 10 (2008) 017.

[49] C. F. Kolda and J. March-Russell, Phys. Rev. D 60, 023504 (1999).

[50] K. Enqvist and J. McDonald, Phys. Rev. Lett. 83, 2510 (1999); Phys. Rev. D 62, 043502 (2000).
[51] M. Kawasaki and F. Takahashi, Phys. Lett. B 516, 388 (2001).

[52] K. W. Ng, Nucl. Phys. B321, 528 (1989).

[53] K. Mukaida and K. Nakayama, J. Cosmol. Astropart. Phys. 01 (2013) 017; 01 (2013) 017.

[54] J. A. Harvey and M. S. Turner, Phys. Rev. D 42, 3344 (1990).

[55] K. A. Olive et al. (Particle Data Group), Chin. Phys. C 38, 090001 (2014).

[56] P. A. R. Ade et al. (Planck Collaboration), arXiv: 1502.02114.

[57] K. Mukaida and M. Yamada, J. Cosmol. Astropart. Phys. 02 (2016) 003.

[58] E. J. Copeland, A. R. Liddle, D. H. Lyth, E. D. Stewart, and D. Wands, Phys. Rev. D 49, 6410 (1994).

[59] G. R. Dvali, Q. Shafi, and R. K. Schaefer, Phys. Rev. Lett. 73, 1886 (1994).

[60] M. Bastero-Gil, S. F. King, and Q. Shafi, Phys. Lett. B 651, 345 (2007).

[61] K. Nakayama, F. Takahashi, and T. T. Yanagida, J. Cosmol. Astropart. Phys. 12 (2010) 010.

[62] W. Buchmuller, L. Covi, and D. Delepine, Phys. Lett. B 491, 183 (2000).

[63] W. Buchmller, V. Domcke, K. Kamada, and K. Schmitz, J. Cosmol. Astropart. Phys. 07 (2014) 054.

[64] S. Nakamura and M. Yamaguchi, Phys. Lett. B 638, 389 (2006); M. Kawasaki, F. Takahashi, and T. T. Yanagida, Phys. Lett. B 638, 8 (2006); T. Asaka, S. Nakamura, and M. Yamaguchi, Phys. Rev. D 74, 023520 (2006); M. Dine, R. Kitano, A. Morisse, and Y. Shirman, Phys. Rev. D 73, 123518 (2006); M. Endo, K. Hamaguchi, and F. Takahashi, Phys. Rev. D 74, 023531 (2006); M. Kawasaki, F. Takahashi, and T. T. Yanagida, Phys. Rev. D 74, 043519 (2006); M. Endo, M. Kawasaki, F. Takahashi, and T. T. Yanagida, Phys. Lett. B 642, 518 (2006).

[65] M. Bolz, A. Brandenburg, and W. Buchmuller, Nucl. Phys. B606, 518 (2001); B790, 336 (2008).

[66] J. Pradler and F. D. Steffen, Phys. Rev. D 75, 023509 (2007).

[67] W. Buchmuller, K. Schmitz, and G. Vertongen, Nucl. Phys. B851, 481 (2011).

[68] M. Kawasaki, K. Kohri, and N. Sugiyama, Phys. Rev. Lett. 82, 4168 (1999); Phys. Rev. D 62, 023506 (2000);

K. Ichikawa, M. Kawasaki, and F. Takahashi, Phys. Rev. D 72, 043522 (2005); M. Kawasaki, K. Kohri, and T. Moroi, Phys. Rev. D 71, 083502 (2005); M. Kawasaki, K. Kohri, T. Moroi, and A. Yotsuyanagi, Phys. Rev. D 78, 065011 (2008).

[69] M. Kawasaki, M. Yamaguchi, and T. Yanagida, Phys. Rev. Lett. 85, 3572 (2000).

[70] R. Kallosh and A. Linde, J. Cosmol. Astropart. Phys. 11 (2010) 011.

[71] M. Kawasaki, M. Yamaguchi, and T. Yanagida, Phys. Rev. D 63, 103514 (2001).

[72] F. Takahashi and M. Yamada, Phys. Lett. B 756, 216 (2016).

[73] K. Harigaya, M. Ibe, M. Kawasaki, and T. T. Yanagida, Phys. Lett. B 756, 113 (2016).

[74] K. Nakayama, F. Takahashi, and T. T. Yanagida, J. Cosmol. Astropart. Phys. 08 (2013) 038. 
[75] N. Arkani-Hamed and S. Dimopoulos, J. High Energy Phys. 06 (2005) 073; G. F. Giudice and A. Romanino, Nucl. Phys. B699, 65 (2004); 706, 65 (2005); N. Arkani-Hamed, S. Dimopoulos, G. F. Giudice, and A. Romanino, Nucl. Phys. B709, 3 (2005).
[76] J. D. Wells, Phys. Rev. D 71, 015013 (2005).

[77] L. J. Hall and Y. Nomura, J. High Energy Phys. 01 (2012) 082.

[78] M. Ibe and T. T. Yanagida, Phys. Lett. B 709, 374 (2012); M. Ibe, S. Matsumoto, and T. T. Yanagida, Phys. Rev. D 85, 095011 (2012). 